\title{
Granular and Star-Shaped Price Systems
}

\author{
Erio Castagnoli', Marzia De Donno², Gino Favero², Paola Modesti2* \\ ${ }^{1}$ Accademia Nazionale Virgiliana and Università Bocconi, Mantova and Milan, Italy \\ ${ }^{2}$ Università degli Studi di Parma Dipartimento di Economia, Parma, Italy \\ Email: paola.modesti@unipr.it
}

Received 17 July 2015; accepted 27 September 2015; published 30 September 2015

Copyright (C) 2015 by authors and Scientific Research Publishing Inc.

This work is licensed under the Creative Commons Attribution International License (CC BY). http://creativecommons.org/licenses/by/4.0/

\section{(c) (7) Open Access}

\begin{abstract}
Linear price systems, typically used to model "perfect" markets, are widely known not to accommodate most of the typical frictions featured in "actual" ones. Since some years, "proportional" frictions (taxes, bid-ask spreads, and so on) are modeled by means of sublinear price functionals, which proved to give a more "realistic" description. In this paper, we want to introduce two more classes of functionals, not yet widely used in Mathematical Finance, which provide a further improvement and an even closer adherence to actual markets, namely the class of granular functionals, obtained when the unit prices of traded assets are increasing w.r.t. the traded amount; and the class of star-shaped functionals, obtained when the average unit prices of traded assets are increasing w.r.t. the traded amount. A characterisation of such functionals, together with their relationships with arbitrages and other (more significant) market inefficiencies, is explored.
\end{abstract}

\section{Keywords}

Arbitrage, Asset Pricing, Super-Hedging, Granularity, Star-Shaped Prices

\section{Introduction}

One of the first and biggest concerns of Mathematical Finance is to study the prices of a suitable set of risky financial assets of any type, including stocks, indexed bonds, variable rate deposits, derivative securities, and so on. Usually, financial assets are modeled as random variables on some state sets, which are supposed to be the same for every asset in the considered market.

In earlier models, such as the one leading to the celebrated Black, Scholes, and Merton formula for option pricing (Black \& Scholes, 1973; Merton, 1973), the market is supposed to be a perfect one; in particular, no frictions are featured and no bid-ask spreads or commissions affect prices. Consequently, as it is clearly shown, for instance, in Dothan (1990), Pliska (1997), and Björk (1999), asset prices turn out to be linear with respect to assets themselves, in the sense that the price of the sum of two positions exactly equals the sum of the two sepa-

${ }^{*}$ Corresponding author. 
rated prices. In such a setting, a central result is found, called the Fundamental Theorem of Asset Pricing, of which dozens of variants are featured in the literature (besides the cited books see, for instance, Delbaen \& Schachermayer, 1994, for quite a general version), which essentially is a representation theorem: roughly speaking, the market prices do not allow for arbitrages (i.e., free gains without risks) if and only if there exist a probability measure called the risk neutral probability - and a discount factor such that prices themselves are (discounted) expected values of the future asset values.

The perfect market model, however, quickly proves to be unfit to provide a good description of realistic markets. For instance, it may be impossible to obtain the exact replication of a given pay-off, and therefore no linear price can be given for it. In such a case, as investigated by Davis \& Clark (1994), the investor may naturally aim at super-hedging it, i.e., at getting at least as much as needed (possibly more) at the minimum possible price. There are also cases when, due to market frictions, a dynamical strategy that exactly replicates the given pay-off may turn out to be more expensive than a super-hedging one: see, e.g., Hodges \& Neuberger (1989). El Karoui \& Quenez (1995), Jouini \& Kallal (1995), Jouini (1997), and Cvitanić et al. (1999) among others, had investigated such a setting and found a representation theorem: in any case, super-hedging prices turn out to be the maximum of a family of linear prices, which, for instance, Cvitanić et al. (1999) interpreted as prices in "shadow markets”. Moreover, Pham (2000) studied the properties of super-hedging price functionals to find them sublinear: additivity was replaced by subadditivity, meaning that the price of the sum of two positions might be cheaper than the sum of the two separated prices. Since sublinearity entails positive homogeneity besides subadditivity, it turns out to be perfectly fit to describe markets affected by proportional transaction costs (such as taxes or percentage commissions): see, e.g., Pham, Touzi, \& Touzi (1999). Furthermore, sublinearity turned out to be interesting for risk management purposes as well, being the foundational point for the celebrated paper by Artzner et al. (1999) on coherent risk measures.

A class of risk measures more general than the sublinear (i.e., coherent) ones of Artzner et al. (1999) is proposed by Föllmer \& Schied (2002), who replace sublinearity with the weaker convexity ${ }^{1}$. Inspired by their work, we started wondering whether convex price functions may sensibly be adapted to financial markets: we realized that this was naturally the case, for instance, if unit asset prices are supposed to increase with respect to the traded amount. A representation result can be found, stating that convex price functionals are the upper envelope of a family of affine prices, which admit an interpretation similar to the "shadow markets" of Cvitanić et al. (1999).

Another, further generalisation, may require average unit asset prices to be increasing, instead of "marginal" ones. This may be the case, for instance, when an agent can choose to buy an asset on several different markets, featuring different increasing unit prices: of course, the purchase will be conducted in such a way that the overall price (or, which is the same, the average unit price) is as low as possible. This leads to a totally new class of price functions, which we name star-shaped because their epigraph turns out to be a star-shaped set with respect to the origin, in the sense of Stewart \& Tall (1983). A representation result can be given for this class of functionals as well, with an interesting economical interpretation.

In the remaining part of this section, the notation used throughout the entire paper is stated, and the current state of the literature about linear and sublinear prices is briefly summarized. Although in different notation, everything exposed here can be found, for instance, in Dothan (1990), Pliska (1997), and Björk (1999) for the linear setting, and in Jouini \& Kallal (1995) and Koehl \& Pham (2000) for the sublinear case. Some examples, in a simple discrete setting, are also given, in order to allow the reader for familiarising with the phenomena under study. Remarkably, we emphasise that, as soon as the price functional is no longer linear, market efficiency is no longer guaranteed by absence of arbitrages only, and that another class of inefficiencies, namely the convenient super-hedgings (roughly speaking, the opportunity to get a better pay-off at a lower price), have to be taken into account.

Section 2 is dedicated to introducing and examining the convex case. After observing that convex functions naturally pop out when pricing by super-hedging by means of assets whose unit price is increasing, we give a generalisation of the Fundamental Theorem of Asset Pricing, and give an interpretation of the representation in

\footnotetext{
${ }^{1}$ Further generalisations of convex risk measures have been proposed. El Karoui \& Ravanelli (2009) remarked that cash additivity, which was a required property of coherent risk measures, should be replaced with cash subadditivity in several cases (e.g., with lack of liquidity for the "riskless" asset). Starting from this, Cerreia-Vioglio et al. (2011) proposed to replace convexity with quasi-convexity in order to better maintain the financial meaning of "diversification" originally connected with the subadditivity requirement. An interpretation of such generalisation in terms of financial prices, though, does not appear to be immediate, and is still under investigation.
} 
terms of market efficiency. It turns out that the market is fully efficient, i.e., that no convenient super-hedging is possible, if all of the "shadow markets" are efficient, whereas absence of arbitrage is guaranteed by a local, less restrictive property.

Star-shaped prices are analysed in Section 3. We show that such functionals are the result of pricing by superhedging by means of assets whose average price is increasing, and show that such a requirement is actually a proper generalisation of the previous, convex case. We also introduce a new pricing technique, which we may call "super-hedging by chunks" and that mathematically corresponds to the inf-convolution of the price functionals of the "shadow markets". We show that convexity and star-shape are in some sense "stable" under super-hedging, either in the classical sense or in the "by chunks" one, and analyse the representation of star-shaped functionals in terms of market efficiency.

Finally, Section 4 is dedicated to summarising and comparing the main properties and the efficiency conditions of the four analysed market types and Section 5 features some concluding remarks.

\subsection{Notation}

A state space $\Omega$ is supposed to be given, and the market $\mathcal{M}$ is a set of (real valued) random variables ${ }^{2}$ $X: \Omega \rightarrow \mathbb{R}:$ every $X \in \mathcal{M}$ is identified with an asset, in the sense that the (random) value attained by $X$ corresponds to the pay-off (or the market value) of the considered asset at a suitable maturity. We are supposing that the uncertainty is resolved in a single time period: in other words, the models we encompass are of a static, not dynamic, type. We write $X \geqq Y$ (respectively, $X>Y$ ) to intend that $X(\omega) \geq Y(\omega)$ (respectively, $X(\omega)>Y(\omega))$ for every $\omega \in \Omega$, where $\geq$ indicates the usual weak inequality between real numbers.

We shall suppose one of the classical "perfect market hypotheses" to hold, requiring every asset $X \in \mathcal{M}$ to be infinitely available (there is no "maximum tradable amount”) and divisible (it is possible to buy any fraction of it); furthermore, short sales are allowed. This translates into the fact that, for every $a \in \mathbb{R}$ and every $X \in \mathcal{M}$, the investor can hold the position $a X$ (where $a<0$ indicates short sale of $|a|$ units of $X$ ). Of course, several assets $X_{1}, X_{2}, \cdots, X_{n}$ can be simultaneously traded, by buying $a_{1}, a_{2}, \cdots, a_{n}$ units of each (with $a_{j}<0$ indicating short sale); this corresponds to holding a portfolio of those $n$ assets, whose "final" pay-off plainly turns out to be $a_{1} X_{1}+a_{2} X_{2}+\cdots+a_{n} X_{n}$. Mathematically speaking, this corresponds to $\mathcal{M}$ being a linear space.

Giving a price to every traded asset $X \in \mathcal{M}$ simply amounts to defining a (price) functional $\pi: \mathcal{M} \rightarrow \mathbb{R}$. The functional $\pi$ is said to allow for:

- An arbitrage (see, e.g., Björk, 1999 and Pliska, 1997) if there exist a $X \geqq 0$ in $\mathcal{M}$ such that $\pi(X)<0$ (which means that it is possible to obtain an immediate gain, corresponding to the negative price, without any risk, i.e., with the certainty not to lose any money at the maturity);

- A convenient super-hedging (quite a recent concept: see, e.g., Castagnoli et al., 2009 and Castagnoli et al., 2011, but also, for instance, Hodges \& Neuberger (1989), who observe the phenomenon although without specifically titling it) if there exist $X, Y \in \mathcal{M}$ such that $X \geqq Y$ and $\pi(X)<\pi(Y)$ (which means that it is possible to obtain a "higher" pay-off at a "lower" price).

Of course, the basic laws of supply and demand imply that neither of the above opportunities, which we shall jointly refer to as inefficiencies, should hold in a market: in both cases, the demand pressure on $X$ would quickly lead its price $\pi(X)$ to increase until becoming either positive (in the first case) or greater than $\pi(Y)$ (in the second case; furthermore, lack of demand on $Y$ would lead its price $\pi(Y)$ to decrease as well). Note that:

- $\pi$ does not allow for arbitrages if and only if $\pi(X) \geq 0$ whenever $X \geqq 0$, that is, if $\pi$ is (or may be called) positive;

- $\pi$ does not allow for convenient super-hedgings if and only if $\pi(X) \geq \pi(Y)$ whenever $X \geqq Y$, that is, if $\pi$ is (or may be called) increasing.

Generally speaking, absence of arbitrages has the nature of a local property, because it only involves the behaviour of the price functional $\pi$ with respect to the null pay-off, whereas absence of convenient super-hedgings is a global property, because it is required to hold for every pair $X, Y \in \mathcal{M}$.

It is noteworthy as well that there are no general links between positivity and monotonicity. Take for instance, $\mathcal{M}=\mathbb{R}^{n}$ : the functional $\pi(y):=\|y\|$ is (of course) positive but not increasing (because, taken a $q<0$ in $\mathbb{R}^{n}$, it is $2 q<q$ and $\|2 q\|>\|q\|)$, whereas, taken a $p>0$ in $\mathbb{R}^{n}$, the functional $\pi(y)=p y-1$ is increasing but

\footnotetext{
${ }^{2} \mathrm{~A}$ puzzled reader, wondering about the measurability of such random variables, will find some clarifications in Remark 1 below.
} 
not positive (because $\pi\left(\frac{1}{2} p\right)=-\frac{1}{2}<0$, although $\frac{1}{2} p>0$ ).

Note also that, for every $X \in \mathcal{M}$, the price functional $\pi$ induces a function $\pi_{X}: \mathbb{R} \rightarrow \mathbb{R}$ defined by $\pi_{X}(\alpha):=\pi(\alpha X)$, which in a natural way can be called the supply and demand function for $X$.

Remark 1. We purposefully decided to avoid measurability issues: in particular, we never mentioned the ( $\sigma$-)algebra $\mathcal{F}$ on $\Omega$ where the probability $P$ is properly defined (and with respect to which all the $X \in \mathcal{M}$ have to be measurable). This is only possible because of our choice of dealing with single period models: in order to introduce a dependence from time, actually, it is unavoidable to follow the well-known approach of defining a filtration $\left(\mathcal{F}_{t}\right)_{t \in \mathcal{T}}$ of ( $\sigma$-)algebrae contained in $\mathcal{F}$ and to suppose that, at every time $t \in T$, the value (price) of a random variable $X \in \mathcal{M}$ is given by its conditional expected value $E\left(X \mid \mathcal{F}_{t}\right)$, possibly discounted in a suitable way.

In the same way, the price functional $\pi: \mathcal{M} \rightarrow \mathbb{R}$ should be taken to be measurable with respect to the ( $\sigma$-)algebra $\mathcal{F}$ (and the Borel $\sigma$-algebra $\mathcal{A}$ on $\mathbb{R}$ ). As a matter of fact, the most general setting for this situation is to take an arbitrary (real) linear space $\mathcal{X}$ and to consider as possible price functionals all of the elements of a subspace $\mathcal{X}^{\prime}$ of the algebraic dual of $\mathcal{X}$. Moreover, by considering on $\mathcal{X}$ the weak topology (i.e., the minimal one that makes continuous all of the $\varphi \in \mathcal{X}^{\prime}$ ), $\mathcal{X}^{\prime}$ turns out to be the topological dual of $\mathcal{X}$, so that our setting can be included in the topological duality among linear spaces, a typical topic in Functional Analysis. Some more details can be found in Castagnoli et al. (in print) and references therein.

Remark 2. The arbitrage and convenient super-hedging opportunities defined above are often called strong in the literature, and their weak counterparts are defined as follows. Write $X \geq Y$ to indicate that $X \geqq Y$ and $X \neq Y$ (that is, there exists at least an $\bar{\omega} \in \Omega$ such that $X(\bar{\omega})>Y(\bar{\omega})$ ). In such a case, the functional $\pi$ is said to allow for:

- A weak arbitrage (or an arbitrage of the second kind) if there exists a $X \geq 0$ in $\mathcal{M}$ such that $\pi(X) \leq 0$ (the case $\pi(X)=0$ is allowed, possibly cancelling the immediate gain, but in some states a gain at the maturity will be obtained);

- A weak convenient super-hedging (or a convenient super-hedging of the second kind) if there exist $X, Y \in \mathcal{M}$ such that $X \geq Y$ and $\pi(X) \leq \pi(Y)$ (the prices may coincide, but in some states $X$ will pay off strictly better than $Y$ ).

It is straightforward that:

- $\pi$ does not allow for weak arbitrages if and only if $\pi(X)>0$ whenever $X \geq 0$ : that is, if and only if $\pi$ is strictly positive;

- $\pi$ does not allow for weak convenient super-hedgings if and only if $\pi(X)>\pi(Y)$ whenever $X \geq Y$ : that is, if and only if $\pi$ is strictly increasing.

As a matter of fact, when the assets $X \in \mathcal{M}$ are not discrete random variables, the above definitions turn out to be impossible to deal with (they would imply, for instance, that for every $\omega \in \Omega$ the "Dirac function" $\delta_{\omega}$ gets a positive price $\pi\left(\delta_{\omega}\right)>0$, which is plainly meaningless). It is then customary to take into consideration an a-priori probability $P$ on $\Omega$, and to define $X \geq Y$ when $X \geqq Y$ and $P\{X>Y\}>0$. In such a case, all of the "inequalities" between random variables are of course to be intended in the " $P$-almost everywhere" sense.

We decided not to take into consideration the weak arbitrages, both for the sake of simplicity and because we want to emphasize that there is no actual need for the $a$ priori probability $P$ to be given. It is nevertheless proper to cite this cases, both for compatibility with the existing literature and to remark that asking for weaker and weaker inefficiencies to be removed from the market translates into stronger and stronger regularity properties for the price functional $\pi$.

It is also noteworthy that, in order to define weak inefficiencies and to intend the inequalities "almost everywhere", instead of an a priori probability $P$, any a priori measure $\lambda$ equivalent to $P$ could be considered on $\Omega$ : it would actually be exactly the same to define $X \geq Y$ whenever $X \geqq Y$ and $\lambda(\{X>Y\})>0$, i.e., when the set where $X>Y$ has a positive measure instead of a positive probability. Briefly said, the normalisation property of the a priori measure is completely unnecessary.

\subsection{Perfect Markets: The Linear Case}

Besides the infinite availability and divisibility hypotheses cited above, the classical models based on "perfect markets” (see the already cited Björk, 1999, Pliska, 1997, and Dothan, 1990) ask for three more requirements. 
First of all, all market agents are fully rational and they aim at maximising their profit; furthermore, all agents are equally informed, without "informational asymmetries". Secondly, the agents are price takers: they have no possibility to negotiate the prices they see on the markets. Finally, in the market there are no taxes, no bid-ask spreads, no commissions: in a word, there are no frictions.

All of these hypotheses together could be simply summarised in a single property: a market is called perfect if the price functional $\pi: \mathcal{M} \rightarrow \mathbb{R}$ is linear. Recall that the linearity of $\pi$ means that $\pi(a X+b Y)=a \pi(X)+b \pi(Y)$ for every $X, Y \in \mathcal{M}$ and every $a, b \in \mathbb{R}$; equivalently, $\pi$ is additive $(\pi(X+Y)=\pi(X)+\pi(Y)$ for every $X, Y \in \mathcal{M})$ and homogeneous $(\pi(a X)=a \pi(X)$ for every $X \in \mathcal{M}$ and every $a \in \mathbb{R}$ ). Note that this translates into the fact that the unit price for every asset $X \in \mathcal{M}$ does not depend on the traded amount: buying (or short selling) $a$ units of $X$ exactly costs (or yields) $|a|$ times the unit price of $X$. In other words, the supply and demand function $\pi_{X}$ is a linear function for every $X \in \mathcal{M}$ : for every $a \in \mathbb{R}, \pi_{X}(\alpha)=\alpha \pi(X)$.

Every linear functional on a linear space attains null value at the "origin" (i.e., at the null vector): as an immediate consequence, an increasing linear functional turns out to be positive as well. Shortly said, for linear functionals, (increasing) monotonicity implies positivity. In the case of linear functionals, moreover, the converse is also true: $X \geqq Y$ is equivalent to $X-Y \geqq 0$, and the fact that $\pi(X-Y)=\pi(X)-\pi(Y)$ immediately yields that a positive linear functional is increasing as well. In other words, positivity and monotonicity are equivalent in the linear setting: therefore, in the classical literature about perfect markets, convenient super-hedgings have never been specifically recognised as market inefficiencies, because a price functional allows for convenient super-hedgings if and only if it allows for arbitrages.

A classical duality result states that, given a linear space $\mathcal{M}$ of real valued functions defined on the same set $\Omega$, a functional $\pi: \mathcal{M} \rightarrow \mathbb{R}$ is linear if and only if there exist a (signed) measure $\mu$ on $\Omega$ such that

$$
\pi(X)=\int_{\Omega} X \mathrm{~d} \mu=\int_{\Omega} X(\omega) \mathrm{d} \mu(\omega) \text { for every } X \in \mathcal{M}
$$

(Lebesgue integrals). Usually, it is said that $\pi$ can be represented as the Lebesgue integral with respect to a suitable measure $\mu$ defined on $\Omega$; we remark that the measure $\mu$ may be "signed", i.e., that it may attain negative values. The Fundamental Theorem of Asset Pricing, translated into our setting, states that the price functional $\pi$ allows for no arbitrages if and only if it is represented by a "proper" positive measure $\mu$.

If the "constant" (degenerate) random variables $a \mathbb{I}_{\Omega}$ belong to $\mathcal{M}$ (or, equivalently, if the monetary unit $\mathbb{I}_{\Omega}$ belongs to $\left.\mathcal{M}\right)$, then the price of $\mathbb{I}_{\Omega}$ amounts to $\pi\left(\mathbb{I}_{\Omega}\right)=\int_{\Omega} 1 \mathrm{~d} \mu=\mu(\Omega)$ : in other words, the "normalisation factor" $B:=\mu(\Omega)$ has the financial meaning of the discount factor for the considered time period. Note also that the measure $Q:=1 / B \cdot \mu$ turns out to be a probability on $\Omega:$ this way, the above representation of the price functional becomes

$$
\pi(X)=\int_{\Omega} X \mathrm{~d} \mu=B \cdot \int_{\Omega} X \mathrm{~d} Q=B \cdot E_{Q}(X),
$$

which is classically told by stating that, if no arbitrages are allowed, the current prices of financial assets are the discounted expected values of their final random pay-off. In such a case, $Q$ is called a risk-neutral probability (or, in the dynamical case, a martingale measure).

Example 1. Take into consideration the state space $\Omega=\left\{\omega_{1}, \omega_{2}\right\}$ : since $\Omega$ is finite, every random variable $X: \Omega \rightarrow \mathbb{R}$ can be identified with the vector $\left[\begin{array}{l}x_{1} \\ x_{2}\end{array}\right]=\left[\begin{array}{l}X\left(\omega_{1}\right) \\ X\left(\omega_{2}\right)\end{array}\right] \in \mathbb{R}^{2}:$ therefore, we shall simply write $X=\left[\begin{array}{l}x_{1} \\ x_{2}\end{array}\right]$. Suppose that two assets are exchanged on the market: $X^{1}=\left[\begin{array}{l}2 \\ 6\end{array}\right]$, at price 4 , and $X^{2}=\left[\begin{array}{l}8 \\ 4\end{array}\right]$, at price 5.

The decision to hold a portfolio obtained by buying (or short selling) $a_{1}$ units of $X^{1}$ and $a_{2}$ units of $X^{2}$ $\left(a_{1}, a_{2} \in \mathbb{R}\right)$ can be identified with the vector $a=\left[\begin{array}{l}a_{1} \\ a_{2}\end{array}\right]:$ it leads to the pay-off $\left[\begin{array}{l}2 a_{1}+8 a_{2} \\ 6 a_{1}+4 a_{2}\end{array}\right]$ and costs $4 a_{1}+5 a_{2}$. Note that every pay-off $\left[\begin{array}{l}x_{1} \\ x_{2}\end{array}\right]$ can be obtained by means of a suitable (and unique) portfolio: in other words, $\mathcal{M}=\mathbb{R}^{2}$, where it is intended that the price of every $X \in \mathbb{R}^{2}$ is defined as the price of the portfolio $a$ 
yielding the pay-off $X$.

We can simplify the notation by defining the pay-off matrix $\quad \boldsymbol{X}=\left[\begin{array}{ll}X^{1} & X^{2}\end{array}\right]=\left[\begin{array}{ll}2 & 8 \\ 6 & 4\end{array}\right]$ : this way, the portfolio $a \in \mathbb{R}^{2}$ simply leads to the pay-off $\boldsymbol{X} a$ (usual matrix product). If we further define the price vector $p=\left[\pi\left(X^{1}\right) \pi\left(X^{2}\right)\right]=[45]$, it is clear that the price of the portfolio $a$ is $p \cdot a$.

Note that every linear functional $\varphi: \mathbb{R}^{2} \rightarrow \mathbb{R}$ simply amounts to the vector ("inner") product by a vector $\left[\begin{array}{ll}\varphi_{1} & \varphi_{2}\end{array}\right] \in \mathbb{R}^{2}$, with $\varphi_{1}:=\varphi\left(\left[\begin{array}{l}1 \\ 0\end{array}\right]\right)$ and $\varphi_{2}:=\varphi\left(\left[\begin{array}{l}0 \\ 1\end{array}\right]\right):$ indeed, $\varphi\left(\left[\begin{array}{l}x_{1} \\ x_{2}\end{array}\right]\right)=\varphi\left(x_{1} \cdot\left[\begin{array}{l}1 \\ 0\end{array}\right]+x_{2} \cdot\left[\begin{array}{l}0 \\ 1\end{array}\right]\right)=x_{1} \varphi_{1}+x_{2} \varphi_{2}=\left[\varphi_{1} \varphi_{2}\right] \cdot\left[\begin{array}{l}x_{1} \\ x_{2}\end{array}\right]$.

Let us now represent the price functional $\pi$. As we already mentioned, for every $X \in \mathbb{R}^{2}$ it has to be $\pi(X)=p a$, with $a$ such that $\boldsymbol{X} a=X$. Suppose now that $\varphi$ is a vector such that $\varphi \boldsymbol{X}=p:^{3}$ it is immediate that $p a=\varphi \boldsymbol{X} a=\varphi X$, and therefore that $\varphi$ represents $\pi$. Since the linear system $\varphi \cdot\left[\begin{array}{ll}2 & 8 \\ 6 & 4\end{array}\right]=\left[\begin{array}{ll}4 & 5\end{array}\right]$ has the unique solution $\varphi=\left[\begin{array}{ll}0.35 & 0.55]\end{array}\right]$, such a vector turns out to be the representation of the linear price functional $\pi$ induced by the market prices.

It is immediate to realise that, since both components of $\varphi$ are positive, the functional $\varphi$ is (positive, and therefore) monotonically increasing. This shows that no arbitrages are allowed in the market. Note also that the discount factor is $B=\varphi \cdot\left[\begin{array}{l}1 \\ 1\end{array}\right]=\varphi_{1}+\varphi_{2}=0.9$, and that the vector $q=\frac{1}{0.9} \cdot \varphi=\left[\frac{7}{18} \frac{11}{18}\right]$ corresponds to a probability $Q$ on $\Omega$, assigning $Q\left(\omega_{1}\right)=\frac{7}{18}, Q\left(\omega_{2}\right)=\frac{11}{18}$. Furthermore, for every $X \in \mathbb{R}^{2}$, $\pi(X)=\varphi \cdot X=B q \cdot X=B E_{Q}(X)$.

Just for the sake of completeness, suppose that the price of $X^{1}$ be 8 instead of 4 . In this case, the unique solution of the system $\varphi \cdot\left[\begin{array}{ll}2 & 8 \\ 6 & 4\end{array}\right]=\left[\begin{array}{l}8 \\ 5\end{array}\right]$ would be $\varphi=[-0.051 .35]$ the presence of a non-positive component implies that $\varphi$ is not positive and that indicates the possibility of arbitrages. Indeed, the pay-off $X=\left[\begin{array}{c}20 \\ 0\end{array}\right] \geqq 0$ is obtained with the portfolio $a=\left[\begin{array}{c}-2 \\ 3\end{array}\right]$ at the price $p a=-1=\varphi X$.

\subsection{Proportional Frictions: The Sublinear Case}

A natural generalisation of the linear model is to suppose that some frictions affect the market, in order to accommodate, for instance, taxes or commissions. By supposing such frictions to be proportional to the traded amount, it is possible to maintain "half" of the homogeneity property of the price functional: namely, $\pi$ turns out to be positively homogeneous, meaning that $\pi(a X)=a \pi(X)$ for every $X \in \mathcal{M}$ and every $a \geq 0$ (no longer for every $a \in \mathbb{R}$ ).

It is clear that such a price functional can no longer be expected to be additive: for instance, an agent buying both $X$ and $-X$ will pay the taxes and commissions on both of them, and thus will end up paying a positive price for the null pay-off: in symbols, $\pi(X)+\pi(-X) \geq \pi(X-X)=0$. Nevertheless, since the agents are still supposed to be rational, it is reasonable to suppose that $\pi$ is subadditive, i.e., that $\pi(X+Y) \leq \pi(X)+\pi(Y)$ (if the price of a joint position were greater than the sum of the two composing ones, every rational agent would separately buy the two components).

If $X \in \mathcal{M}$, generally speaking, the bid price induced by a pricing functional is $\pi_{b}(X):=-\pi(-X)$. If $\pi$ is

${ }^{3}$ It is natural to wonder whether such a $\varphi$ always exists. It can be shown (see Pliska, 1997 and Castagnoli et al., 2009) that existence is guaranteed by a weaker condition than the absence of arbitrages, called the law of one price (roughly speaking, there is no way to obtain the same pay-off at two different prices): this always happens if, as in this example, the pay-off of the listed assets are linearly independent. 
(sublinear, and therefore) subadditive, recalling that $\pi(X)+\pi(-X) \geq 0$, we get

$\pi_{b}(X)=-\pi(-X) \leq \pi(X)=\pi_{a}(X)$, where we write $\pi_{a}$ to underline that those expressed by $\pi$ actually are ask prices. Roughly speaking, then, sublinear functionals model the case when the ask and the bid price may differ (due to taxes, commissions, or general bid-ask spreads), yet the unit price does not depend on the traded amount. The supply and demand function induced by a sublinear $\pi$ for a given $X \in \mathcal{M}$ takes the form

$\pi_{X}(\alpha)=\left\{\begin{array}{ll}\alpha \pi_{a}(X) & \alpha \geq 0 \\ \alpha \pi_{b}(X) & \alpha<0\end{array}\right.$.

Every sublinear functional attains null value at the origin: therefore, every increasing sublinear functional is positive as well. The converse is not true, as already mentioned: the norm functional is positive, but not increasing. As a consequence, there may be sublinear price functionals that allow for convenient super-hedgings although not allowing for arbitrages. It is noteworthy, nevertheless, that $\pi$ turns out to be increasing every time that it is "negative", i.e., when $\pi(Y) \leq 0$ for every $Y \leqq 0$ : in such a case, indeed, whenever $X \geqq Y$ we get $\pi(Y)=\pi(X+Y-X) \leq \pi(X)+\pi(Y-X) \leq \pi(X)$. Recalling that the bid price of the pay-off $X$ is naturally defined as $\pi_{b}(X)=-\pi(-X)$, the "negativity" condition translates into $\pi_{b}(X) \geq 0$ for every $X \geqq 0$ : in other words, absence of arbitrages is guaranteed by the positivity of ask prices of the positive pay-offs, whereas absence of convenient super-hedgings is ensured by the positivity of bid prices of the same positive pay-offs.

As an immediate consequence of the classical Hahn-Banach Theorem, a sublinear functional $\pi$ can be represented as the pointwise maximum of the linear functionals that it "dominates". In greater detail: if $\pi$ is a sublinear functional, then the set $L:=\{\varphi: \mathbb{R} \rightarrow \mathbb{R}: \varphi$ linear, $\varphi \leqq \pi\}$ is not empty and $\pi(X)=\sup _{\varphi \in L} \varphi(X)$. Moreover, if $\pi$ is not allowed to take infinite values, $L$ turns out to be convex and compact, so that the "sup "can be replaced by a "max". It is possible to show (see Pliska, 1997 and Castagnoli et al., 2009) that:

- $\pi$ is positive if and only if there exists (at least) a positive $\varphi \in L$;

- $\pi$ is increasing if and only if every $\varphi \in L$ is positive.

From a mathematical point of view, $L$ is the subdifferential of $\pi$ at 0 (see, e.g., Rockafellar, 1970).

According to such a characterisation, if $\pi$ does not allow for convenient super-hedgings (and, therefore, not even for arbitrages), every $\varphi \in L$ can be represented as the expected value with respect to a suitable measure $Q_{\varphi}$, discounted by a suitable factor $B_{\varphi}$ :

$$
\pi(X)=\max _{\varphi \in L}\left[B_{\varphi} \cdot E_{Q_{\varphi}}(X)\right] .
$$

In other words, an efficient sublinear functional acts "as if" a whole set $L$ of "plausible" scenarios $\varphi$ are involved, each corresponding to (a linear price functional, i.e., to) a probability measure $Q_{\varphi}$ and a discount factor $B_{\varphi}$ : the price assigned to every random variable amounts to the "worst case" discounted expected value, i.e., to the linear functional assigning the highest price to $X$. It is noteworthy to mention that such a representation was already conjectured by de Finetti \& Obry (1933).

One final consideration is in order. A rational investor who aims at obtaining the pay-off $Z: \Omega \rightarrow \mathbb{R}$ (which need not belong to $\mathcal{M}$ ) is naturally led to look for the best (super)hedge of $Z$, i.e., to buy the cheapest traded asset $X \in \mathcal{M}$ that dominates $Z$ (El Karoui \& Quenez, 1995). This way, (a better pay-off than) $Z$ can be obtained at the price

$$
\tilde{\pi}(Z)=\min \{\pi(X): X \in \mathcal{M}, X \geqq Z\},
$$

called the cheapest super-hedging price of $Z$. It is quite clear that, if $\pi$ does not allow for convenient super-hedgings, $\tilde{\pi}(X)=\pi(X)$ for every $X \in \mathcal{M}$; on the other hand, it is immediate to realise that, if $\pi$ allows for convenient super-hedgings, then $\tilde{\pi} \leq \pi$. It is indeed possible to show that $\tilde{\pi}$ is sublinear as soon as $\pi$ is and that, if $\pi(\cdot)=\max \{\varphi(\cdot): \varphi \in L\}$, then $\tilde{\pi}(\cdot)=\max \left\{\varphi(\cdot): \varphi \in L_{+}\right\}$with $L_{+}:=\{\varphi \in L: \varphi$ is positive $\}$. Roughly speaking, $\tilde{\pi}$ turns out to be the highest sublinear functional, among those dominated by $\pi$, that does not allow for (arbitrages or) convenient super-hedgings.

Example 2. Consider the same two assets of Example 1, with pay-off matrix $\boldsymbol{X}=\left[\begin{array}{ll}2 & 8 \\ 6 & 4\end{array}\right]$, but suppose now that two price vectors are given, namely that of the ask prices $p_{a}=\left[\begin{array}{ll}4 & 5\end{array}\right]$ and of the bid prices $p_{b}=\left[\begin{array}{ll}3.6 & 4.4\end{array}\right]$ 
(of course $p_{b} \leqq p_{a}$ ). The price of every $X \in \mathbb{R}^{2}$ is found as its cheapest super-hedging price: $\pi(X)=\min \left\{4 a_{1}^{+}-3.6 a_{1}^{-}+5 a_{2}^{+}-4.4 a_{1}^{-}: a_{1} X^{1}+a_{2} X^{2} \geqq X\right\} \quad$ (where $a^{+}:=\max \{a, 0\}$ and $a^{-}:=\max \{-a, 0\}$ denote the positive and negative part of $a \in \mathbb{R}$ respectively). A standard linear programming duality argument ${ }^{4}$ allows to conclude that the price $\pi$ dominates the linear functional induced by the vector $\varphi=\left[\begin{array}{ll}\varphi_{1} & \varphi_{2}\end{array}\right]$ if and only if $p_{b} \leq \varphi \boldsymbol{X} \leq p_{a}$, which amounts to finding all the $\varphi$ s such that $\varphi \boldsymbol{X}=\vartheta p_{a}+(1-\vartheta) p_{b}=\left[\begin{array}{ll}3.6+0.4 \vartheta & 4.4+0.6 \vartheta\end{array}\right]$, with $0 \leq \vartheta \leq 1$.

The solutions of the given parametric linear system is the set $\left.L=\left\{\begin{array}{ll}0.3+0.05 \vartheta & 0.5+0.05 \vartheta\end{array}\right]: 0 \leq \vartheta \leq 1\right\}:$ it is immediate to check that it is a convex and compact subset of $\mathbb{R}^{2}$. Since $L$ contains positive vectors only, we can conclude that $\pi$ allows for no convenient super-hedgings (and, therefore, for no arbitrages).

It is possible to build examples when the functional $\pi$ induced by the listed assets allows for arbitrages and for convenient super-hedgings, or for convenient super-hedgings only. For the sake of brevity, we invite the interested reader to see Castagnoli et al. (2009). ${ }^{5}$

\section{Increasing Unit Prices}

\section{The Granular (Convex) Case}

Although sublinear prices can indeed capture several features of prices in the "real world", they still feature unit prices which do not depend on the traded amount. Who trades on actual markets, instead, knows well that unit prices tend to increase with respect to the amount bought, and to decrease with respect to the amount sold. Suppose, for instance, that we are set to buy 1000 units of some asset. Having a look at the offer prices, we see that someone is selling up to 100 units at $3 €$ each, someone else up to 500 units at $3.1 €$ each, someone else up to 600 units at $3.2 €$ each, and so on. This way, we are facing increasing unit prices, and to buy all of the 1000 units we have to pay $3 \times 100+3.1 \times 500+3.2 \times 400=3130 €$ : it is immediate to realise that, generally speaking, total price needed to buy $\alpha>0$ units of an asset turns out to be a(n increasing and) convex function of $\alpha$ (and piecewise affine, in our example, but this is not necessary: the price is a convex function of the traded amount every time that the marginal price is increasing, which is the standard hypothesis of the classical law of supply and demand). We want to show that a natural way to model such a situation is to take into consideration a convex price functional $\pi: \mathcal{M} \rightarrow \mathbb{R}$ (which is of course a generalisation of the sublinear case, because every sublinear functional is convex as well): in order to do so, let us see how a convex price functional comes out in a very natural way.

Suppose that, in an exchange list under consideration, the assets $X^{1}, X^{2}, \cdots, X^{n}$ are included, such that, for every $j=1,2, \cdots, n$, the supply and demand function $\pi_{X^{j}}: \alpha \rightarrow \pi\left(\alpha X^{j}\right)$ is increasing and convex. Of course, the set $\mathcal{M}$ of all attainable pay-offs is the linear space spanned by the traded assets:

$\mathcal{M}=\left\{\alpha_{1} X^{1}+\alpha_{2} X^{2}+\cdots+\alpha_{n} X^{n}: \alpha_{1}, \alpha_{2}, \cdots, \alpha_{n} \in \mathbb{R}\right\}$. The only reasonable way of assigning a price to every $Y \in \mathcal{M}$ is to use the super-hedging technique seen at the end of the previous section:

$$
\pi(Y)=\min \left\{\sum_{j=1}^{n} \pi\left(\alpha_{j} X^{j}\right): \sum_{j=1}^{n} \alpha_{j} X^{j} \geqq Y\right\} .
$$

It is immediate to show that such functional $\pi$ is increasing ${ }^{5}$; since $\pi(0)=0$, the monotonicity of $\pi$ implies its positivity, which, from the financial point of view, means that the "cheapest super-hedging" price functional $\pi$ does not allow neither for arbitrages nor for convenient super-hedgings. It is also possible, although a

\footnotetext{
${ }^{4}$ The dual of the problem $\begin{aligned} & \min p_{a} a^{+}-p_{a} a^{-} \\ & \operatorname{sub} \boldsymbol{X}\left(a^{+}-a^{-}\right)=Y\end{aligned} \quad \begin{aligned} & \min \varphi Y \\ & \text { is } \begin{array}{c}p_{a} \geqq \varphi \boldsymbol{X} \\ -p_{a} \geqq-\varphi \boldsymbol{X}\end{array}\end{aligned}$, which can be written as $\begin{aligned} & \min \varphi Y \\ & \text { sub } p_{b} \leqq \varphi \boldsymbol{X} \leqq p_{a}\end{aligned}$; the dual of

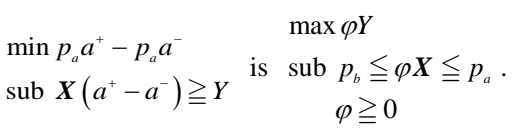

${ }^{5}$ Suppose that $Y, Z \in \mathcal{M}$ are such that $Y \geqq Z$. This means that every portfolio that super-hedges $Y$ super-hedges $Z$ : therefore, the min that corresponds to the super-hedging price $\pi(Y)$ is evaluated on a subset of the set where the min corresponding to $\pi(Z)$ is evaluated. As a consequence, $\pi(Y) \geq \pi(Z)$.
} 
little technical, to show that $\pi$ is convex, i.e., that $\pi(\vartheta X+(1-\vartheta) Y) \leq \vartheta \pi(X)+(1-\vartheta) \pi(Y)$ for every $X, Y \in \mathcal{M}$ and every $\vartheta \in[0,1]^{6}$ : the convexity of the single supply and demand functions "propagates" to the entire pricing functional.

Fenchel's Theorem ensures that a convex functional $\pi$ can be represented as the pointwise maximum of the affine functionals that it dominates, where an affine functional is the translation of a linear functional: $f: \mathcal{M} \rightarrow \mathbb{R}$ is affine if there exist $\varphi: \mathcal{M} \rightarrow \mathbb{R}$ linear and $c \in \mathbb{R}$ such that $f(\cdot)=\varphi(\cdot)+c$. In greater detail:

Proposition 1 (Fenchel's Theorem). Let $\pi: \mathcal{M} \rightarrow \mathbb{R}$ be convex. Then the set

$L:=\{f: \mathcal{M} \rightarrow \mathbb{R}: f$ affine, $f \leqq \pi\}$ is non-empty, closed and convex and such that $\pi(X)=\max _{f \in L} f(X)$ for every $X \in \mathcal{M}$.

Since every $f \in L$ can be written as $\varphi_{f}+c_{f}$ with $\varphi_{f}: \mathcal{M} \rightarrow \mathbb{R}$ linear and $c_{f} \in \mathbb{R}$, and since every linear functional can be represented as in (1), the convex functional $\pi$ can be represented as

$$
\pi(X)=\max _{f \in L}\left[\varphi_{f}(X)+c_{f}\right]=\max _{f \in L}\left[B_{f} \mathrm{E}_{Q_{f}}(X)+c_{f}\right] \quad(X \in \mathcal{M}) .
$$

Note that $\pi(0)=0$ implies that all of the constants $c_{f}$ are $\leq 0$, and that at least one of them is null, because $\pi(0)=\max _{f \in L}\left[\varphi_{f}(0)+c_{f}\right]=\max _{f \in L} c_{f}$.

Example 3. Take into consideration the same two assets of Examples 1 and 2, with pay-off matrix, $\boldsymbol{X}=\left[\begin{array}{ll}2 & 8 \\ 6 & 4\end{array}\right]$, and suppose that they are exchanged on the market as follows:

- $X^{1}$ has unit price 4 for (short) sales or purchases up to 10 units, 4.2 for purchases up to 50 units and 4.4 beyond 50 units;

- $X^{2}$ has unit price 5 up to 20 units, 5.5 up to 80 units and 6 beyond 80 units.

Such prices split the portfolio space into nine regions, identified by four vertices (see Figure 1).

- $a^{1}=\left[\begin{array}{l}10 \\ 20\end{array}\right]$, which yields the pay-off $\boldsymbol{X} a^{1}=\left[\begin{array}{l}180 \\ 140\end{array}\right]$ and costs $4 \times 10+5 \times 20=140$;

- $a^{2}=\left[\begin{array}{l}10 \\ 80\end{array}\right]$, which yields the pay-off $\boldsymbol{X} a^{2}=\left[\begin{array}{l}660 \\ 380\end{array}\right]$ and costs $4 \times 10+5 \times 20+5.5 \times 60=470$ (recall the

first 20 units of $X^{2}$ are bought at the cheaper price 5, and only the 60 subsequent units are bought at the higher price 5.5;

- $a^{3}=\left[\begin{array}{l}50 \\ 20\end{array}\right]$, which yields the pay-off $\boldsymbol{X} a^{3}=\left[\begin{array}{l}260 \\ 380\end{array}\right]$ and costs $4 \times 10+4.2 \times 40+5 \times 20=308$;

- $a^{4}=\left[\begin{array}{l}50 \\ 80\end{array}\right]$, which yields the pay-off $\boldsymbol{X} a^{4}=\left[\begin{array}{l}740 \\ 620\end{array}\right]$ and costs $4 \times 10+4.2 \times 40+5 \times 20+5.5 \times 60=638$.

Inside each region, the unit prices $p^{i}$ remain constant (shown in Figure 1 as well), and therefore the price functional $\pi$ is affine: we may write $\pi_{\mid \mathcal{A}_{i}}=f^{i}=\varphi^{i}+c_{i}, \quad i=1,2, \cdots, 9$.

In greater detail: there have to be nine vectors $\varphi^{1}, \varphi^{2}, \cdots, \varphi^{9} \in \mathbb{R}^{2}$ and nine (non positive) constants

${ }^{6}$ To prove the inequality, suppose that the cheapest (super) hedges for $Y, Z \in \mathcal{M}$ are, respectively, $\sum_{j=1}^{n} \beta_{j} X^{j}$ and $\sum_{j=1}^{n} \gamma_{j} X^{j}$, so that $\pi(Y)=\sum_{j=1}^{n} \pi\left(\beta_{j} X^{j}\right)$ and $\pi(Z)=\sum_{j=1}^{n} \pi\left(\gamma_{j} X^{j}\right)$. Let $0 \leq \vartheta \leq 1$, and consider the portfolio $W=\sum_{j=1}^{n}\left(\vartheta \beta_{j}+(1-\vartheta) \gamma_{j}\right) X^{j}$. Of course, $W \geqq \vartheta Y+(1-\vartheta) Z$; therefore, $\pi(\vartheta Y+(1-\vartheta) Z) \leq \pi(W)$.

Since $\pi(W)$ is defined as the cheapest (super)hedge of $W, \pi(W) \leq \sum_{j=1}^{n} \pi\left(\left[\vartheta \beta_{j}+(1-\vartheta) \gamma_{j}\right] X^{j}\right)$. On the other hand, the fact that all of the functions $\pi_{x^{j}}(j=1,2, \cdots, n)$ are convex w.r.t. $\alpha$ implies that

$\sum_{j=1}^{n} \pi\left(\left[\vartheta \beta_{j}+(1-\vartheta) \gamma_{j}\right] X^{j}\right) \leq \sum_{j=1}^{n}\left[\vartheta \pi\left(\beta_{j} X^{j}\right)+(1-\vartheta) \pi\left(\gamma_{j} X^{j}\right)\right]=\vartheta \pi(Y)+(1-\vartheta) \pi(Z)$. By transitivity,

$\pi(\vartheta Y+(1-\vartheta) Z) \leq \vartheta \pi(Y)+(1-\vartheta) \pi(Z)$, i.e., $\pi$ is convex.

Note that this implies that the inequalities (3) hold for every $X \in \mathcal{M}$, not just for the listed assets. 


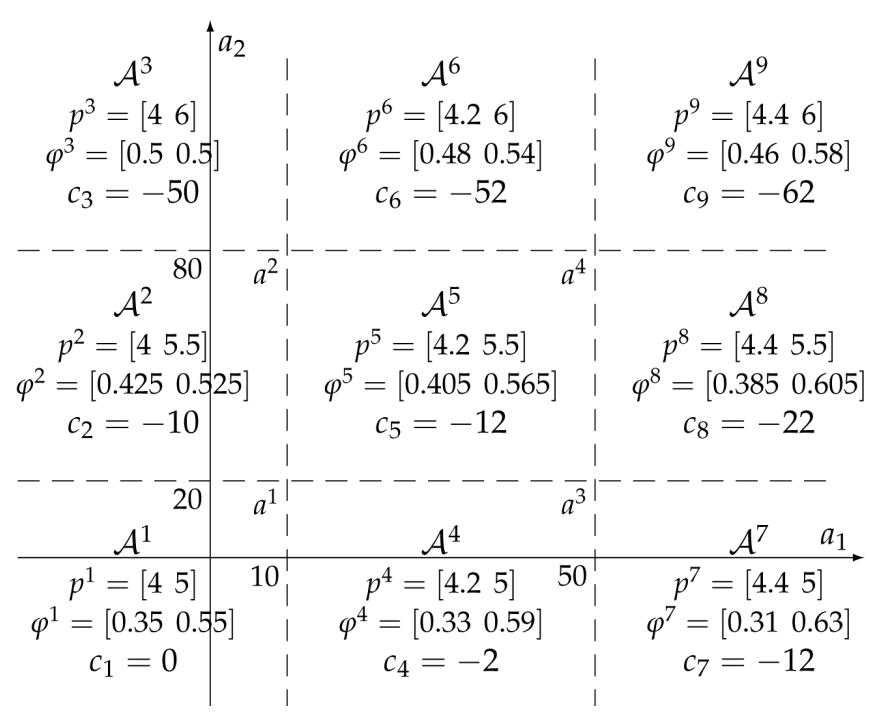

Figure 1. The portfolio space in Example 3.

$c_{1}, c_{2}, \cdots, c_{n} \in \mathbb{R}$ such that, if $X=\boldsymbol{X} a$ with $a \in \mathcal{B} \quad(j=1,2, \cdots, 9)$, then $\pi(X)=\varphi^{j} X+c_{j}$. Every vector $\varphi^{j}$ $(j=1,2, \cdots, 9)$ identifies a discount factor $B_{j}$ and a risk-neutral probability $Q_{j}$, and therefore this model identifies at least nine risk-neutral measures; however, as already pointed out, the risk-neutral measures turn out not to be as important as the properties of the price functional in order to investigate market efficiency.

Note that, if both $X$ and $X+H$ belong to the same region $\mathcal{A}_{j}$, then $\pi(X+H)-\pi(X)=\varphi^{j} H$ : it is then straightforward to realise that, for every $j=1,2, \cdots, n$, the vector $\varphi^{j}$ is easily determined by solving the usual linear system $\varphi^{j} \boldsymbol{X}=p^{j}$. The constants $c_{j}, j=1,2, \cdots, n$, are calculated as the amount "saved" by buying the "first" units at a price smaller than $p^{j}$ :

- In $\mathcal{A}_{1}$, the effective prices are the lowest ones: therefore, $c_{1}=0$ (we could argue the same conclusion from the fact that $\left.0=\pi(0)=\varphi^{1} \cdot 0+c_{1}\right)$;

- In $\mathcal{A}_{2}$, the price of $X^{2}$ is 5.5, but the first 20 units are bought at the price $5=5.5-0.5$, thus "saving" $20 \times 0.5=10$ : therefore, $c_{2}=-10$ (as a double check, consider for instance that the portfolio $a=\left[\begin{array}{c}5 \\ 20\end{array}\right] \in \mathcal{A}_{2}$ yields the pay-off $\boldsymbol{X} a=\left[\begin{array}{l}410 \\ 230\end{array}\right]$ and costs $5 \times 4+20 \times 5+(50-20) \times 5.5=285$, and $285=\varphi^{2} \cdot\left[\begin{array}{l}410 \\ 230\end{array}\right]+c_{2}$ precisely yields $c_{2}=-10$ );

- In $\mathcal{A}_{3}$, the price of $X_{2}$ is 6 , but the first 20 units are bought at $6-5=1$ less and the subsequent $(80-20=) 60$ at $6-5.5=0.5$ less, for a total "saving" of $20 \times 1+60 \times 0.5=40$ : therefore, $c_{3}=-50$ (note that such a saving can also be calculated as the one achieved in the "previous" region $\mathcal{A}_{2}$, i.e., $20 \times 0.5=10$, plus the additional saving of 0.5 on all of the first 80 units: $20 \times 0.5+80 \times 0.5=50$ );

- In $\mathcal{A}_{4}$, the price of $X^{1}$ is 4.2 , but the first 10 units are bought at $4.2-4=0.2$ less, thus "saving" $10 \times 0.2=2$ : therefore, $c_{4}=-2$;

- In $\mathcal{A}_{5}$, both the first 10 units of $X^{1}$ and the first 20 units of $X^{2}$ are bought at a lower price: the savings of $\mathcal{A}_{2}$ and $\mathcal{A}_{4}$ add up, and therefore $c_{5}=c_{2}+c_{4}=-12$.

- In $\mathcal{A}_{6}$, the savings of $\mathcal{A}_{3}$ and $\mathcal{A}_{4}$ add up, and therefore $c_{6}=c_{3}+c_{4}=-52$;

- In $\mathcal{A}_{7}$, the first 10 units of $X^{1}$ cost $4.4-4=0.4$ less than the "full" price, and the subsequent $(50-10=)$ 40 cost $4.4-4.2=0.2$ less: the total saving is $10 \times 0.4+40 \times 0.4=12$ : therefore, $c_{7}=-12$;

- In $\mathcal{A}_{8}$, the savings of $\mathcal{A}_{2}$ and $\mathcal{A}_{7}$ add up, and therefore $c_{8}=c_{2}+c_{7}=-22$;

- Finally, $c_{9}=c_{3}+c_{7}=-62$.

Now, the price of every pay-off $X \in \mathbb{R}^{2}$ can be calculated as $\pi(X)=\max _{j=1,2, \cdots, 9}\left[\varphi^{j} X+c_{j}\right]$. For instance, for $X=\left[\begin{array}{c}800 \\ 1200\end{array}\right]$ we get: 


\begin{tabular}{c|c|c|c}
$j$ & $\varphi^{j} \cdot X$ & $c_{j}$ & $\varphi^{j} X+c_{j}$ \\
\hline 1 & $0.35 \cdot 800+0.55 \cdot 1200=940$ & -0 & 940 \\
2 & $0.425 \cdot 800+0.525 \cdot 1200=970$ & -10 & 960 \\
3 & $0.5 \cdot 800+0.5 \cdot 1200=1000$ & -50 & 950 \\
4 & $0.33 \cdot 800+0.59 \cdot 1200=972$ & -2 & 970 \\
5 & $0.405 \cdot 800+0.565 \cdot 1200=1002$ & -12 & 990 \\
6 & $0.48 \cdot 800+0.54 \cdot 1200=1032$ & -52 & 980 \\
7 & $0.31 \cdot 800+0.63 \cdot 1200=1004$ & -12 & 992 \\
8 & $0.385 \cdot 800+0.605 \cdot 1200=1034$ & -22 & $\mathbf{1 0 1 2}$ \\
9 & $0.46 \cdot 800+0.58 \cdot 1200=1064$ & -62 & 1002
\end{tabular}

(the maximum price is emphasised). Note that, indeed, $X$ is yielded by the portfolio $a=\left[\begin{array}{c}160 \\ 60\end{array}\right] \in \mathcal{A}_{8}$, and the price of $a$ is $10 \times 4+40 \times 4.2+110 \times 4.4+20 \times 5+40 \times 5.5=1012$.

As already mentioned, the price functional $\pi$ is convex. We want nevertheless to strike out that, generally speaking, it is neither sub- nor superadditive: for instance, consider again the pay-off $X=\left[\begin{array}{c}800 \\ 1200\end{array}\right]$. It is possible to check that $\pi\left(\left[\begin{array}{c}800 \\ 0\end{array}\right]\right)=350$ and $\pi\left(\left[\begin{array}{c}0 \\ 1200\end{array}\right]\right)=744$, and therefore $\pi\left(\left[\begin{array}{c}800 \\ 1200\end{array}\right]\right)=1012<1094=\pi\left(\left[\begin{array}{c}800 \\ 0\end{array}\right]\right)+\pi\left(\left[\begin{array}{c}0 \\ 1200\end{array}\right]\right)$. On the other hand, it is also $\pi\left(\left[\begin{array}{c}400 \\ 600\end{array}\right]\right)=495$, and therefore $\pi\left(\left[\begin{array}{c}800 \\ 1200\end{array}\right]\right)=1012>990=\pi\left(\left[\begin{array}{l}400 \\ 600\end{array}\right]\right)+\pi\left(\left[\begin{array}{l}400 \\ 600\end{array}\right]\right)$. We want to point out that this second inequality does not correspond to a convenient super-hedging: indeed, it is not possible to buy simultaneously two portfolios yielding the claim $\left[\begin{array}{l}400 \\ 600\end{array}\right]$ because, when doubling the position, the unit prices of the traded assets increase.

It is still possible to show that, if $\pi$ does not take infinite values, the set $\Phi_{L}:=\left\{\varphi_{f}: f \in L\right\}$ is compact and convex. Mathematically speaking, the set $\Phi_{L}$ is the union of the sub differentials of $\pi$ at all points $X \in \mathcal{M}$; note that, if $\pi$ is sublinear, then $\Phi_{L}=L$.

In perfect analogy to what happens for sublinear functionals, $\pi$ is increasing if and only if every $\varphi \in \Phi_{L}$ is positive. The natural technique of pricing any pay-off $Z$ (either belonging to $\mathcal{M}$ or not) by super-hedging, as seen in Section 1.3, can still be applied, even in the case when $\pi$ allows for convenient super-hedgings, and it can be shown that the cheapest super-hedging price $\tilde{\pi}$ is a convex functional if the "original" $\pi$ is. Furthermore, the set $\Phi_{\tilde{L}}$ corresponding to the set $\tilde{L}$ identified by $\tilde{\pi}$ turns out to be precisely the set $\left(\Phi_{L}\right)_{+}=\left\{\varphi \in \Phi_{L}: \varphi \geqq 0\right\}$.

Example 4. On $\Omega=\left\{\omega_{1}, \omega_{2}\right\}$ consider the two assets:

- $X^{1}=\left[\begin{array}{l}4 \\ 5\end{array}\right]$, at the unit price of 4.5 , which increases at 4.7 for purchases of more than 10 units;

- $X^{2}=\left[\begin{array}{l}3 \\ 4\end{array}\right]$, traded at 3.4 per unit, which increases at 3.7 for purchases of more than 20 units.

This way, $\boldsymbol{X}=\left[\begin{array}{ll}4 & 3 \\ 5 & 4\end{array}\right]$. The prices split the portfolio space into four regions $\mathcal{A}_{1}, \mathcal{A}_{2}, \mathcal{A}_{3}, \mathcal{A}_{4}$, corresponding to the four "quadrants" identified by the portfolio $\bar{a}=\left[\begin{array}{l}10 \\ 20\end{array}\right]$ (see Table 1); note that the portfolio $\bar{a}$ yields the pay-off $\left[\begin{array}{l}100 \\ 130\end{array}\right]$ and costs $10 \times 4.5+20 \times 3.4=113$. The vectors $\varphi_{j}$ and the constants $c_{j}$, calculated as in 
Table 1. The four regions of the portfolio space in Example 4.

\begin{tabular}{c|c|c|c|c}
$\mathcal{A}_{j}$ & portfolios & \multicolumn{1}{|c|}{$p^{j}$} & \multicolumn{1}{|c}{$\varphi^{j}$} & $c_{j}$ \\
\hline $\mathcal{A}_{1}$ & $a_{1} \leqslant 10, a_{2} \leqslant 20$ & $p^{1}=\left[\begin{array}{ll}4.5 & 3.4\end{array}\right]$ & $\varphi^{1}=\left[\begin{array}{ll}1 & 0.1\end{array}\right]$ & $c_{1}=0$ \\
$\mathcal{A}_{2}$ & $a_{1} \leqslant 10, a_{2} \geqslant 20$ & $p^{2}=\left[\begin{array}{ll}4.5 & 3.7\end{array}\right]$ & $\varphi^{2}=\left[\begin{array}{ll}-0.5 & 1.3\end{array}\right]$ & $c_{2}=-6$ \\
$\mathcal{A}_{3}$ & $a_{1} \geqslant 10, a_{2} \leqslant 20$ & $p^{3}=\left[\begin{array}{ll}4.7 & 3.4\end{array}\right]$ & $\varphi^{3}=\left[\begin{array}{ll}1.8 & -0.5\end{array}\right]$ & $c_{3}=-2$ \\
$\mathcal{A}_{4}$ & $a_{1} \geqslant 10, a_{2} \geqslant 20$ & $p^{4}=\left[\begin{array}{ll}4.7 & 3.7\end{array}\right]$ & $\varphi^{4}=\left[\begin{array}{ll}0.3 & 0.7\end{array}\right]$ & $c_{4}=-8$
\end{tabular}

previous Example 3, are also shown.

There are positive vectors in $\Phi_{L}$ (such as $\varphi^{1}$, for instance); yet, the negative components of $\varphi^{2}$ and $\varphi^{3}$ indicate the possibility of a convenient super-hedging. It is quite clear that such possibilities apply to all of the portfolios belonging to the regions $\mathcal{A}_{2}$ and $\mathcal{A}_{3}$.

Consider, for instance, the portfolio $a^{2}=\left[\begin{array}{ll}-5 & 30\end{array}\right] \in \mathcal{A}_{2}$, whose pay-off is $\boldsymbol{X} \boldsymbol{a}^{2}=\left[\begin{array}{l}70 \\ 95\end{array}\right]$ and whose price is $-5 \times 4.5+20 \times 3.4+10 \times 3.7=82.5=\left[\begin{array}{ll}-0.5 & 1.3\end{array}\right] \cdot\left[\begin{array}{l}70 \\ 95\end{array}\right]-6$. It is immediate to check that the pay-off $\boldsymbol{X} a^{2}=\left[\begin{array}{l}70 \\ 95\end{array}\right]$ can be super-hedged by means of the portfolio $\tilde{a}^{2}=\left[\begin{array}{c}3 \\ 20\end{array}\right] \in \mathcal{A}_{1}\left(\cap \mathcal{A}_{2}\right)$, whose pay-off is $\left[\begin{array}{c}72 \\ 95\end{array}\right]$ and whose price is $3 \times 4.5+20 \times 3.4=81.5<82.5$ : a convenient super-hedging is found, and the cheapest super-hedging price functional $\tilde{\pi}$ will be such that $\tilde{\pi}\left(\left[\begin{array}{l}110 \\ 145\end{array}\right]\right) \leq 81.5$ (indeed, it can be shown that the equality holds).

Analogously, the portfolio $a^{3}=\left[\begin{array}{l}25 \\ 10\end{array}\right] \in \mathcal{A}_{3} \quad$ yields the pay-off $\quad \boldsymbol{X} a^{3}=\left[\begin{array}{l}130 \\ 165\end{array}\right]$ at the price $10 \times 4.5+15 \times 4.7+10 \times 3.4=149.5=\left[\begin{array}{cc}1.8 & -0.5\end{array}\right] \times\left[\begin{array}{l}130 \\ 165\end{array}\right]-2$, but a convenient super-hedging is given by $\tilde{a}_{3}=\left[\begin{array}{c}17.5 \\ 20\end{array}\right] \in \mathcal{A}_{4}\left(\cap \mathcal{A}_{3}\right)$, whose pay-off is $\left[\begin{array}{c}130 \\ 167.5\end{array}\right]$ and whose price is $10 \times 4.5+7.5 \times 4.7+20 \times 3.4=148.25$. Therefore, $\tilde{\pi}\left(\left[\begin{array}{l}130 \\ 165\end{array}\right]\right) \leq 148.25$ (and, as before, the equality holds, indeed).

It is possible to prove ${ }^{7}$ that the "adjusted" functional $\tilde{\pi}$, calculated after exploiting all of the convenient super-hedgings, is calculated as the maximum of six affine functionals as shown in Table 2. Notably enough, the convex set $\tilde{\Phi}$ identified by the six vectors $\tilde{\varphi}^{1}, \tilde{\varphi}^{2 a}, \cdots, \tilde{\varphi}^{4}$ exactly amounts to the subset of the positive vectors contained in the "original" set $\Phi$ (see Figure 2). Note also that not all of the vectors $\varphi^{j}, j=1,2,3,4$, induce risk neutral measures, because some of them have negative components; however, each of the six "vertices" of the "restricted" set $\tilde{\Phi}$ can again be seen as the product of a risk neutral measure and a discount factor (for instance, $\tilde{\varphi}^{2 \mathrm{~b}}$ corresponds to the degenerate probability $Q^{2 \mathrm{~b}}$ such that $Q^{2 \mathrm{~b}}\left(\left\{\omega_{2}\right\}\right)=1$ and to the discount fac-

${ }^{7}$ We confine ourselves to a few hints, in order to help a reader possibly interested in finding a method into this madness. In the region $\mathcal{A}_{2}$, the "marginal" pay-off $\left[\begin{array}{l}1 \\ 0\end{array}\right]$ (yielded by the "marginal" portfolio $\left[\begin{array}{c}4 \\ -5\end{array}\right]$ ) has a negative cost (indeed, $4 \times 4.5+(-5) \times 3.7=-0.5$ ): therefore the idea is to add to any portfolio $a^{2}=\left[\begin{array}{l}a_{1}^{2} \\ a_{2}^{2}\end{array}\right] \in \mathcal{A}_{2}$ a multiple of $\left[\begin{array}{c}4 \\ -5\end{array}\right]$ until "hitting" the boundary with another region. If $4\left(a_{2}^{2}-20\right) \leq-5\left(a_{1}^{2}-10\right)$, such a region turns out to be $\mathcal{A}_{1}$, and the affine pricing functional used to price the pay-off becomes $\tilde{\varphi}^{2 \mathrm{a}}-\tilde{c}_{2 \mathrm{a}}$; in the opposite case when $4\left(a_{2}^{2}-20\right) \geq 5\left(a_{1}^{2}-10\right)$, the region hit is $\mathcal{A}_{4}$, and the "adjusted" pricing functional is $\tilde{\varphi}^{2 \mathrm{~b}}-\tilde{c}_{2 \mathrm{~b}}$. Analogous considerations apply to the region $\mathcal{A}_{3}$, where the "marginal arbitrage" is the pay-off $\left[\begin{array}{l}0 \\ 1\end{array}\right]$, (yielded by the marginal portfolio $\left[\begin{array}{c}-3 \\ 4\end{array}\right]$ ), which costs -0.5 . 


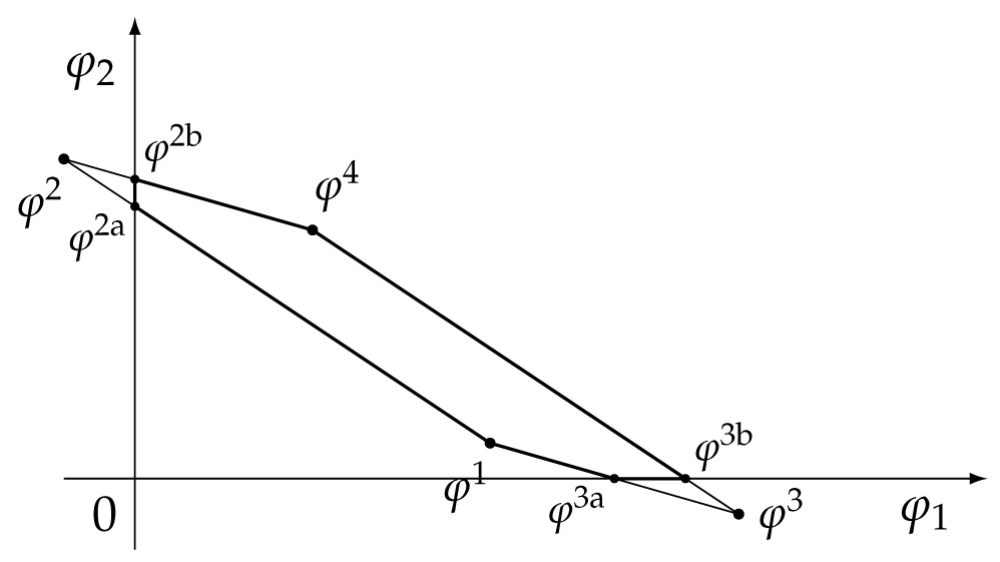

Figure 2. The sets $\Phi$ (thin line) and $\tilde{\Phi}$ (thick line) in Example 4.

Table 2. The four regions of the portfolio space in Example 4 after taking advantage of the convenient super-hedging opportunities.

\begin{tabular}{c|c|c|c}
$\mathcal{A}_{j}$ & portfolios & $\widetilde{\varphi}^{j}$ & $\widetilde{c}_{j}$ \\
\hline $\mathcal{A}_{1}$ & $a_{1} \leqslant 10, a_{2} \leqslant 20$ & $\widetilde{\varphi}^{1}=\left[\begin{array}{ll}1 & 0.1\end{array}\right]$ & $\widetilde{c}_{1}=0$ \\
$\mathcal{A}_{2 \mathrm{a}}$ & $\left\{\begin{array}{l}a_{1} \leqslant 10, a_{2} \geqslant 20 \\
5 a_{1}+4 a_{2} \leqslant 130\end{array}\right.$ & $\widetilde{\varphi}^{2 \mathrm{a}}=\left[\begin{array}{ll}0 & 0.9\end{array}\right]$ & $\widetilde{c}_{2 \mathrm{a}}=-4$ \\
$\mathcal{A}_{2 \mathrm{~b}}$ & $\left\{\begin{array}{c}a_{1} \leqslant 10, a_{2} \geqslant 20 \\
5 a_{1}+4 a_{2} \geqslant 130\end{array}\right.$ & $\widetilde{\varphi}^{2 \mathrm{~b}}=\left[\begin{array}{ll}0 & 0.925\end{array}\right]$ & $\widetilde{c}_{2 \mathrm{~b}}=-7.25$ \\
$\mathcal{A}_{3 \mathrm{a}}$ & $\left\{\begin{array}{c}a_{1} \geqslant 10, a_{2} \leqslant 20 \\
4 a_{1}+3 a_{2} \leqslant 100\end{array}\right.$ & $\widetilde{\varphi}^{3 \mathrm{a}}=\left[\begin{array}{ll}\frac{17}{15} & 0\end{array}\right]$ & $\widetilde{c}_{3 \mathrm{a}}=-\frac{1}{3}$ \\
$\mathcal{A}_{3 \mathrm{~b}}$ & $\left\{\begin{array}{c}a_{1} \geqslant 10, a_{2} \leqslant 20 \\
4 a_{1}+3 a_{2} \geqslant 100\end{array}\right.$ & $\widetilde{\varphi}^{3 \mathrm{~b}}=\left[\begin{array}{ll}1.175 & 0\end{array}\right]$ & $\widetilde{c}_{3 \mathrm{~b}}=-4.5$ \\
$\mathcal{A}_{4}$ & $\begin{array}{c}a_{1} \geqslant 10, a_{2} \geqslant 20 \\
\widetilde{\varphi}^{4}=\left[\begin{array}{ll}0.3 & 0.7\end{array}\right]\end{array}$ & $\widetilde{c}_{4}=-8$
\end{tabular}

tor $\left.B_{2 \mathrm{~b}}=0.925\right)$. Nevertheless, we remark that such elements are not as important as the $\varphi s$ themselves to investigate market efficiency.

When it comes to positivity, things get a little more complicated: indeed, the fact that $\Phi$ contains no positive functionals at all is still sufficient, but no longer necessary, in order to allow for arbitrages. Consider the following (and quite minimal) example.

Example 5. Again on $\Omega=\left\{\omega_{1}, \omega_{2}\right\}$, suppose that the asset $X^{1}=\left[\begin{array}{l}1 \\ 0\end{array}\right]$ is sold at the unit price 0.4 regardless of the amount, and that $X^{2}=\left[\begin{array}{l}0 \\ 1\end{array}\right]$ is sold at unit price -0.1 up to 5 units, and 0.5 for more than 5 units. The portfolio space is trivially split into two regions (and in each of them, since $\boldsymbol{X}=\left[\begin{array}{ll}1 & 0 \\ 0 & 1\end{array}\right]$, it is trivially $\left.\varphi^{j}=p^{j}\right):$

\begin{tabular}{c|c|c|c}
$\mathcal{A}_{j}$ & portf.s & $p^{j}=\varphi^{j}$ & $c_{j}$ \\
\hline $\mathcal{A}_{1}$ & $a_{1} \leqslant 5$ & $p^{1}=\varphi^{1}=\left[\begin{array}{ll}0.4 & -0.1\end{array}\right]$ & $c_{1}=0$ \\
$\mathcal{A}_{2}$ & $a_{1} \geqslant 5$ & $p^{2}=\varphi^{2}=\left[\begin{array}{ll}0.4 & 0.5\end{array}\right]$ & $c_{2}=-3$
\end{tabular}


It is clear that arbitrages are possible, because buying $\left[\begin{array}{l}0 \\ k\end{array}\right]$ with $k \geq 0$ has a negative price for every $k<6$. Nevertheless, the set $\Phi$ contains the positive vector $\varphi^{2}=\left[\begin{array}{ll}0.4 & 0.5\end{array}\right]$.

It is still possible to define the cheapest super-hedging price functional $\tilde{\pi}$ : it turns out that it simply amounts to replace, in the region $\mathcal{A}_{1}, \varphi^{1}$ and $c_{1}$ with $\tilde{\varphi}^{1}=\left[\begin{array}{ll}0.4 & 0\end{array}\right]$ and $\tilde{c}_{1}=-3$. As a consequence, it is no longer $\tilde{\pi}(0)=0$ : indeed, $\tilde{\pi}(0)=-3$, which precisely indicates the possibility to get a free gain of 3 without risk. It is also worth pointing out that such an arbitrage is just "local” in the spirit of Castagnoli et al. (2011), in the sense that there is an upper bound to the gains that can be obtained by means of arbitrages.

The point is that, as already mentioned, an arbitrage is nothing but a convenient super-hedging of the null vector. In the sublinear case, positive homogeneity ensures that such a convenient super-hedging (meaning both its positive pay-off and its negative price) can be multiplied by an arbitrary positive constant and still remain an arbitrage: this way, if arbitrages are possible, the region of the arbitrage portfolios is always unbounded. In the "granular" convex case, instead, positive homogeneity no longer holds, and therefore arbitrages may be confined to a bounded region, as it happens in Example 5.

As a matter of fact, it is possible to show that a linear functional $\varphi \in \Phi$ matters in determining whether $\pi$ allows for arbitrages or not only if $\varphi$ is relative to a "region" of the portfolio space that contains the null pay-off: we call $\Phi_{0}$ such a subset of $\Phi^{8}$. In other terms, while $\Phi$ is the union of all subdifferentials of $\pi$, we are here only interested in the subdifferential $\Phi_{0}$ of $\pi$ at the origin. Briefly, a convex functional $\pi$ is positive if and only if there exists a positive $\varphi \in \Phi_{0}$.

For sublinear functionals, it can be proven that the subdifferential at each point is by necessity a subset of the subdifferential at 0 , or, in other words that $\Phi=\Phi_{0}$. This, besides the "unbounded" nature of arbitrages in sublinear markets, provides a further argument in favour of the fact that, unlike what happens for convex markets, in sublinear markets absence of arbitrages and absence of convenient super-hedgings are properties of the same set $L$.

The results of this section can be summarized and formalized in the following

Theorem 1. Let $\mathcal{M}$ be a linear space of financial assets, and $\pi: \mathcal{M} \rightarrow \mathbb{R}$ be a convex pricing functional such that $\pi(0)=0$. Define $L:=\{f: \mathcal{M} \rightarrow \mathbb{R}: f$ affine, $f \leqq \pi\}$ and, for every $f \in L$, call $c_{f}:=f(0)$ and $\varphi_{f}(\cdot):=f(\cdot)-c_{f}$, so that $f(\cdot)=\varphi_{f}(\cdot)+c_{f}$. Let $\Phi:=\left\{\varphi_{f}: f \in L\right\}$ and $\Phi_{0}:=\{\varphi: \mathcal{M} \rightarrow \mathbb{R}: \varphi$ linear, $\varphi \leqq \pi\}$. Then:

1. $L$ is non-empty, closed and convex;

2. for every $X \in \mathcal{M}, \pi(X)=\max _{f \in L} f(X)$;

3. for every $f \in L, \quad c_{f} \leq 0$ and $\varphi_{f}$ is linear; furthermore, there exists $\bar{f} \in L$ such that $c_{\bar{f}}=0$;

4. $\Phi$ and $\Phi_{0}$ are non-empty, closed and convex, and $\Phi_{0}=L \bigcap \Phi$;

5. $\pi$ is increasing if and only if every $\varphi_{f} \in \Phi$ is positive;

6. $\pi$ is positive if and only if there exists a positive $\varphi \in \Phi_{0}$.

\section{Increasing Average Prices}

\section{The Star-Shaped Case}

Suppose that $X \in \mathcal{M}$ is such that the supply and demand function $\alpha \rightarrow \pi(\alpha X)$ is convex. If, as it is natural to suppose, $\pi(0)=0$, then it turns out that

$$
\begin{array}{ll}
\pi(\alpha X) \leq \alpha \pi(X) & \text { if } 0 \leq \alpha \leq 1, \\
\pi(\alpha X) \geq \alpha \pi(X) & \text { if } \alpha \geq 1
\end{array}
$$

the first inequality comes from the convexity property, because (being $0 \leq \alpha \leq 1$ ) it is

\footnotetext{
${ }^{8}$ In the case when $\pi(0)=0, \Phi_{0}$ can also be defined as the subset of linear (not just affine) functionals of $\Phi$ or, equivalently, as the set of all $\varphi_{f}$ such that $c_{f}=0$.
} 
$\pi(\alpha X+(1-\alpha) 0) \leq \alpha \pi(X)+(1-\alpha) \pi(0)$; it is furthermore possible to see that the two inequalities are equivalent to each other'.

A possible reason why inequalities (3) are sensible in ordinary markets can be seen as follows. Take $\alpha, \beta \in \mathbb{R}$ such that $1 \leq \alpha \leq \beta$, so that $\beta / \alpha>1$. The first of the two inequalities (3) above is equivalent to

$$
\pi(\beta X)=\pi\left(\frac{\beta}{\alpha} \cdot \alpha X\right) \geq \frac{\beta}{\alpha} \pi(\alpha X) \quad \text { i.e., } \frac{\pi(\beta X)}{\beta} \geq \frac{\pi(\alpha X)}{\alpha}:
$$

in other words, the supply and demand function of an $X \in \mathcal{M}$ satisfies (3) if and only if the average unit price of $X$ is increasing with respect to the traded amount. This is why we deem reasonable such a property: indeed, when aiming at purchasing some quantity of something, it is rational to buy it at the lowest possible overall price, which of course coincides with the lowest average unit price.

Suppose, for instance, that three agents sell the same asset $X$ on the market. The first one sells it at 4 per unit, but can only provide up to 30 units. The second one sells it at 5 per unit (for any amount). The third one sells it at 4.5 per unit, but only for a minimum order of 50 units. It is clear that the best price that can be obtained to buy $\alpha>0$ units of $X$ are:

$$
\pi(\alpha X)=\left\{\begin{array}{lc}
4 \alpha & \alpha \leq 30 \\
5 \alpha-30 & 30<\alpha<60 \\
4.5 \alpha & \alpha \geq 60
\end{array}\right.
$$

for instance, to buy 50 units of $X$, the unit price of 4.5 may be obtained, but it is less expensive to buy 30 units from the first agent and 20 from the second, at a total price of $30 \times 4+20 \times 5=220$ instead of $50 \times 4.5=225$. Note that the average price obtained with the "separate" purchase is $220 / 50=4.4<4.5$.

We shall call star-shaped a supply and demand function $\alpha \rightarrow \pi(\alpha X)$ that satisfies inequalities (3) and, in general, any function $f: \mathcal{X} \rightarrow \mathbb{R}$, with $\mathcal{X}$ any real linear space, that does the same. Note the difference with "granular" pricing functionals, which feature an increasing marginal price with respect to the traded amount: of course every convex function is star-shaped as well, but the converse need not be true.

Example 6. The function $f: \mathbb{R} \rightarrow \mathbb{R}$ defined as

$$
f(x)= \begin{cases}0.3 x & x \leq 10 \\ 2 x-1 & x>10\end{cases}
$$

is star shaped, because ( $f(0)=0$ and) its "average value" $f(x) / x$ is increasing. Nevertheless, $f$ is not convex (and not even continuous).

A geometrical interpretation of the star-shaped property is easily deduced from the monotonicity of average prices. Recall that, given any real linear space $\mathcal{X}$, a function $f: \mathcal{X} \rightarrow \mathbb{R}$ is convex if and only if, whenever two points $\left[\begin{array}{c}X^{1} \\ y_{1}\end{array}\right],\left[\begin{array}{c}X^{2} \\ y_{2}\end{array}\right]$ are given "above" the "graph" of $f$, i.e., such that $f\left(X^{j}\right) \leq y_{j} \quad(j=1,2)$, then the entire segment adjoining $\left[\begin{array}{l}X^{1} \\ y_{1}\end{array}\right]$ and $\left[\begin{array}{c}X^{2} \\ y_{2}\end{array}\right]$ remains "above" the graph of $f$ (which translates into $f\left(\vartheta X^{1}+(1-\vartheta) X^{2}\right) \leq \vartheta y_{1}+\left(1-\vartheta y_{2}\right)$ for every $\left.\vartheta \in[0,1]\right)$. The property that average prices are increasing for star-shaped functions translates into the fact that whenever $\left[\begin{array}{c}X \\ y\end{array}\right]$ is given "above" the "graph" of $f$, i.e., such that $f(X) \leq y$, then the entire segment adjoining $\left[\begin{array}{l}0 \\ 0\end{array}\right]$ and $\left[\begin{array}{l}x \\ y\end{array}\right]$ remains "above" the graph of $f$ (which translates into $f(\vartheta X) \leq \vartheta y$ for every $\vartheta \in[0,1])$. In Figure 3 the typical appearance of the four functions examined in this paper is depicted for functions $f: \mathbb{R} \rightarrow \mathbb{R}$.

${ }^{9}$ Define, indeed $\beta=\frac{1}{\alpha}$ and $Y=\alpha X$ : it is clear that $0 \leq \alpha \leq 1$ (respectively, $\alpha>1$ ) if and only if $\beta>1$ (respectively, $0 \leq \beta \leq 1$ ) and that $\pi(\alpha X) \leq \alpha \pi(X)$ can be written as $\pi(Y) \leq \alpha \pi(\beta Y)$, i.e. , $\pi(\beta X) \geq \beta \pi(X)$. 


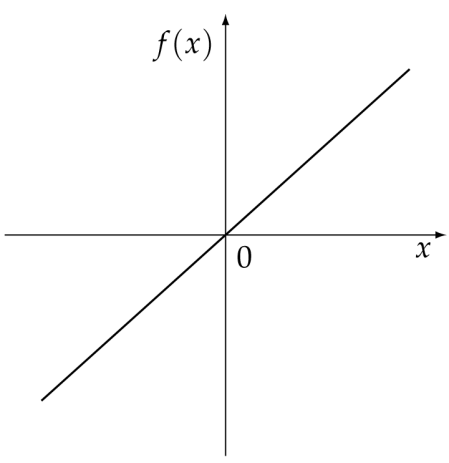

(a)

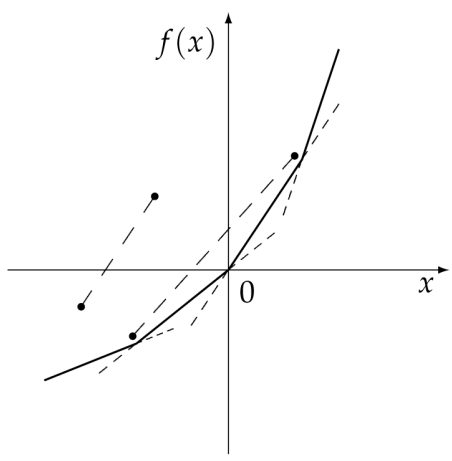

(c)

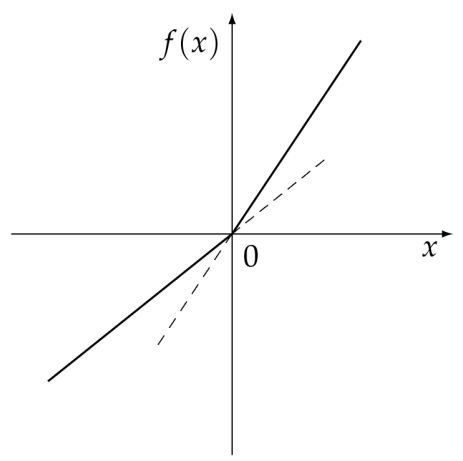

(b)

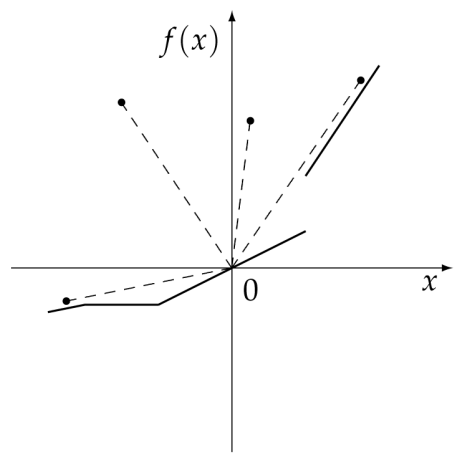

(d)

Figure 3. Typical graphs of (a) a linear function; (b) a sublinear (and not linear) function; (c) a convex (and not sublinear) function and (d) a star-shaped (and neither convex nor continuous) function.

Suppose that, in an exchange list under consideration, the assets $X^{1}, X^{2}, \cdots, X^{n}$ are included, such that, for every $j=1,2, \cdots, n$, the supply and demand function $\alpha \rightarrow \pi\left(\alpha X^{j}\right)$ is increasing and star-shaped. Once again, we define on the set $\mathcal{M}=\left\{\alpha_{1} X^{1}+\alpha_{2} X^{2}+\ldots+\alpha_{n} X^{n}: \alpha_{1}, \alpha_{2}, \cdots, \alpha_{n} \in \mathbb{R}\right\}$ of all attainable pay-offs a price functional $\pi$ by super-hedging:

$$
\pi(Y)=\min \left\{\sum_{j=1}^{n} \pi\left(\alpha_{j} X^{j}\right): \sum_{j=1}^{n} \alpha_{j} X^{j} \geqq Y\right\} .
$$

As usual, such a functional immediately turns out to be increasing (and therefore positive, because $\pi(0)=0$ ); moreover, it can be proved that $\pi$ is star shaped as well, i.e., that inequalities (3) hold for every $X \in \mathcal{M}$. So to say, the star-shape of the single supply and demand functions "propagates" by super-hedging.

An adaptation of a result by Chateauneuf \& Aouani (2008), still unpublished (see Castagnoli et al., 2009), shows that a star-shaped functional $\pi$ can be represented as the pointwise minimum of the convex functions that dominate it. In detail:

Proposition 2. Let $\pi: \mathcal{M} \rightarrow \mathbb{R}$ be a star-shaped functional. Then the set $G:=\{g: \mathcal{M} \rightarrow \mathbb{R}: g$ convex, $g \geqq \pi\} \quad$ is closed and convex, and $\pi(X)=\min _{g \in G} g(X)$ for every $X \in \mathcal{M}$.

It is indeed possible to prove that only the convex functionals $g \in G$ such that $g(0)=0$ can be taken into consideration, i.e., that if $G_{0}:=\{g \in G: g(0)=0\}$, then ( $G_{0}$ is closed and convex as well and) $\pi(X)=\min _{g \in G_{0}} g(X)$ as well. By applying Proposition 1, we can write each $g \in G_{0}$ as in (2), and therefore represent the star-shaped functional $\pi$ as

$$
\pi(X)=\min _{g \in G_{0}} \max _{f_{g} \in L_{g}}\left[\varphi_{f_{g}}(X)+c_{f_{g}}\right] .
$$


To give an economical interpretation of such a representation, think that several agents are available to sell $X$ on the market. Each agent, corresponding to a $g \in G$, assigns a convex price to $X$ (i.e., has an increasing marginal price), and we are free to choose the agent we want to buy the asset $X$ from (i.e., the cheapest one).

It is worth mentioning that the class of star-shaped functionals is closed under pointwise sup and inf, even for an infinite family of functionals; more explicitly, if $\mathcal{X}$ is any real linear space and $\left(f_{s}: \mathcal{X} \rightarrow \mathbb{R}\right)_{s \in S}$ is a family of star-shaped functionals such that $f_{s}=\min _{G_{s}} g$, then $\min _{s} f_{s}=\min _{\cup_{s} G_{s}} g$ and $\max _{s} f_{s}=\min _{\cap_{s} G_{s}} g$.

Example 7. Take into consideration the same two assets of Examples 1, 2, and 3, with pay-off matrix $\boldsymbol{X}=\left[\begin{array}{ll}2 & 8 \\ 6 & 4\end{array}\right]$ We suppose that the investor can call her/his demands on three different markets, each run by a (representative) agent with her/his own convex price system: this way, three convex price functionals $\pi_{1}, \pi_{2}$ and $\pi_{3}$ are given. Suppose that the first one is the same of Example 2, with nine portfolio region as follows:

\begin{tabular}{|c|c|c|c|c|}
\hline $\mathcal{A}_{1, j}$ & portfolios & $p_{1}^{\prime}$ & $\varphi_{1}^{\jmath}$ & $c_{1, j}$ \\
\hline $\mathcal{A}_{1,1}$ & $\begin{array}{l}a_{1} \leqslant 10 \\
a_{2} \leqslant 20\end{array}$ & $p_{1}^{1}=\left[\begin{array}{ll}4 & 5\end{array}\right]$ & $\varphi_{1}^{1}=\left[\begin{array}{ll}0.35 & 0.55\end{array}\right]$ & $c_{1,1}=0$ \\
\hline $\mathcal{A}_{1,2}$ & $\begin{array}{c}a_{1} \leqslant 10 \\
20 \leqslant a_{2} \leqslant 80\end{array}$ & $p_{1}^{2}=\left[\begin{array}{ll}4 & 5.5\end{array}\right]$ & $\varphi_{1}^{2}=\left[\begin{array}{ll}0.425 & 0.525\end{array}\right]$ & $c_{1,2}=-10$ \\
\hline $\mathcal{A}_{1,3}$ & $\begin{array}{l}a_{1} \leqslant 10 \\
a_{2} \geqslant 80\end{array}$ & $p_{1}^{3}=\left[\begin{array}{ll}4 & 6\end{array}\right]$ & $\varphi_{1}^{3}=\left[\begin{array}{ll}0.5 & 0.5\end{array}\right]$ & $c_{1,3}=-50$ \\
\hline $\mathcal{A}_{1,4}$ & $\begin{array}{c}10 \leqslant a_{1} \leqslant 50 \\
a_{2} \leqslant 20\end{array}$ & $p_{1}^{4}=\left[\begin{array}{ll}4.2 & 5\end{array}\right]$ & $\varphi_{1}^{4}=\left[\begin{array}{ll}0.33 & 0.59\end{array}\right]$ & $c_{1,4}=-2$ \\
\hline $\mathcal{A}_{1,5}$ & $\begin{array}{l}10 \leqslant a_{1} \leqslant 50 \\
20 \leqslant a_{2} \leqslant 80\end{array}$ & $p_{1}^{5}=\left[\begin{array}{ll}4.2 & 5.5\end{array}\right]$ & $\varphi_{1}^{5}=\left[\begin{array}{ll}0.405 & 0.565\end{array}\right]$ & $c_{1,5}=-12$ \\
\hline $\mathcal{A}_{1,6}$ & $\begin{array}{c}10 \leqslant a_{1} \leqslant 50 \\
a_{2} \geqslant 80\end{array}$ & $p_{1}^{6}=\left[\begin{array}{ll}4.2 & 6\end{array}\right]$ & $\varphi_{1}^{6}=\left[\begin{array}{ll}0.48 & 0.54\end{array}\right]$ & $c_{1,6}=-52$ \\
\hline $\mathcal{A}_{1,7}$ & $\begin{array}{l}a_{1} \geqslant 50 \\
a_{2} \leqslant 20\end{array}$ & $p_{1}^{7}=\left[\begin{array}{ll}4.4 & 5\end{array}\right]$ & $\varphi_{1}^{7}=\left[\begin{array}{ll}0.31 & 0.63\end{array}\right]$ & $c_{1,7}=-12$ \\
\hline $\mathcal{A}_{1,8}$ & $\begin{array}{c}a_{1} \geqslant 50 \\
20 \leqslant a_{2} \leqslant 80\end{array}$ & $p_{1}^{8}=\left[\begin{array}{ll}4.4 & 5.5\end{array}\right]$ & $\varphi_{1}^{8}=\left[\begin{array}{ll}0.385 & 0.605\end{array}\right]$ & $c_{1,8}=-22$ \\
\hline $\mathcal{A}_{1,9}$ & $\begin{array}{l}a_{1} \geqslant 50 \\
a_{2} \geqslant 80\end{array}$ & $p_{1}^{9}=\left[\begin{array}{ll}4.4 & 6\end{array}\right]$ & $\varphi_{1}^{9}=\left[\begin{array}{ll}0.46 & 0.58\end{array}\right]$ & $c_{1,9}=-62$ \\
\hline
\end{tabular}

The second agents gives the granular price functional $\pi_{2}$ identified by ${ }^{10}$

\begin{tabular}{|c|c|c|c|c|}
\hline $\mathcal{A}_{2, j}$ & portfolios & $p_{1}^{j}$ & $\varphi_{1}^{\gamma}$ & $c_{2, j}$ \\
\hline $\mathcal{A}_{2,1}$ & $\begin{array}{l}a_{1} \leqslant 30 \\
a_{2} \leqslant 30\end{array}$ & $p_{1}^{1}=\left[\begin{array}{ll}4.1 & 5.1\end{array}\right]$ & $\varphi_{1}^{1}=\left[\begin{array}{ll}0.355 & 0.565\end{array}\right]$ & $c_{2,1}=0$ \\
\hline $\mathcal{A}_{2,2}$ & $\begin{array}{l}a_{1} \leqslant 30 \\
a_{2} \geqslant 30\end{array}$ & $p_{1}^{2}=\left[\begin{array}{ll}4.1 & 6.1\end{array}\right]$ & $\varphi_{1}^{2}=\left[\begin{array}{ll}0.505 & 0.515\end{array}\right]$ & $c_{2,2}=-30$ \\
\hline $\mathcal{A}_{2,3}$ & $\begin{array}{l}a_{1} \geqslant 30 \\
a_{2} \leqslant 30\end{array}$ & $p_{1}^{3}=\left[\begin{array}{ll}4.5 & 5.1\end{array}\right]$ & $\varphi_{1}^{3}=\left[\begin{array}{ll}0.315 & 0.645\end{array}\right]$ & $c_{2,3}=-12$ \\
\hline $\mathcal{A}_{2,4}$ & $\begin{array}{l}a_{1} \geqslant 30 \\
a_{2} \geqslant 30\end{array}$ & $p_{1}^{4}=\left[\begin{array}{ll}4.5 & 6.1\end{array}\right]$ & $\varphi_{1}^{4}=\left[\begin{array}{ll}0.465 & 0.595\end{array}\right]$ & $c_{2,4}=-42$ \\
\hline
\end{tabular}

The third agent simply gives a linear price: $p^{3}=\left[\begin{array}{ll}4.2 & 6\end{array}\right], \varphi^{3}=\left[\begin{array}{ll}0.48 & 0.54\end{array}\right]$.

Let us take into consideration some random variables: the details of the calculations (which amount to see in which region the price of the given pay-offs is maximum) are left to the reader.

- For $Y^{1}=\left[\begin{array}{l}300 \\ 300\end{array}\right]$, it is $\pi_{1}\left(Y^{1}\right)=279, \pi_{2}\left(Y^{1}\right)=276$, and $\pi_{3}\left(Y^{1}\right)=306$ : therefore, $Y^{1}$ is bought from the second agent and $\pi\left(Y^{1}\right)=276$.

- For $Y^{2}=\left[\begin{array}{l}180 \\ 140\end{array}\right]$, it is $\pi_{1}\left(Y^{2}\right)=140, \pi_{2}\left(Y^{2}\right)=143$, and $\pi_{3}\left(Y^{2}\right)=162$ : therefore, $Y^{2}$ is bought from the first agent and $\pi\left(Y^{1}\right)=140$.

- For $Y^{3}=\left[\begin{array}{l}5000 \\ 1000\end{array}\right]$, it is $\pi_{1}\left(Y^{3}\right)=2950, \pi^{2}\left(Y^{3}\right)=3010$ and $\pi_{3}\left(Y^{3}\right)=2940$ : therefore, $Y^{3}$ is bought from the third agent and $\pi\left(Y^{3}\right)=2940$.

\footnotetext{
${ }^{10}$ We are supposing here that the three "markets" share the exchange list, i.e., that the same assets are exchanged on the three markets. This is just chosen in order to avoid further complications: indeed, on different markets, different assets could be quoted and exchanged.
} 
It is then clear that each of the three price systems has a chance to prove the cheapest one and, therefore, that it is effective in determining $\pi$ as the pointwise minimum of $\pi_{1}, \pi_{2}$, and $\pi_{3}$. This can happen even for a single asset: for instance, the three demand functions $\alpha \rightarrow \pi(\alpha X) \quad(\alpha \geq 0)$ for $X=\left[\begin{array}{l}17 \\ 16\end{array}\right]$ in the three markets turn out to be:

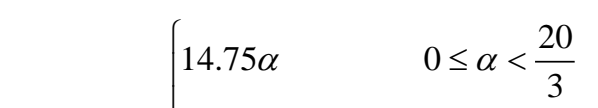

$$
\begin{aligned}
& \pi_{1}(\alpha X)=\left\{\begin{array}{ll}
15.05 \alpha-2 & \frac{20}{3} \leq \alpha<\frac{80}{7} \\
15.925 \alpha-12 & \frac{80}{7} \leq \alpha<\frac{100}{3} \\
16.225 \alpha-22 & \frac{100}{3} \leq \alpha<\frac{320}{7} \\
17.1 \alpha-62 & \alpha \geq \frac{320}{7}
\end{array},\right. \\
& \pi_{2}(\alpha X)= \begin{cases}15.075 \alpha & 0 \leq \alpha<\frac{120}{7} \\
16.825 \alpha-30 & \frac{120}{7} \leq \alpha<20 \\
17.425 \alpha-42 & \alpha \geq 20\end{cases}
\end{aligned}
$$

and $\pi_{3}(\alpha X)=16.8 \alpha$. It is straightforward (but very laboured) to see that

$$
\pi(\alpha X)=\left\{\begin{array}{ll}
\pi_{1}(X) & 0 \leq \alpha<\frac{240}{17} \\
\pi_{2}(X) & \frac{240}{17} \leq \alpha<20 \\
\pi_{1}(X) & 20 \leq \alpha<\frac{620}{3} \\
\pi_{3}(X) & \alpha \geq \frac{620}{3}
\end{array}= \begin{cases}14.75 \alpha & 0 \leq \alpha<\frac{20}{3} \\
15.05 \alpha-2 & \frac{20}{3} \leq \alpha<\frac{80}{7} \\
15.925 \alpha-12 & \frac{80}{7} \leq \alpha<\frac{240}{17} \\
15.075 \alpha & \frac{240}{17} \leq \alpha<\frac{120}{7} \\
16.825 \alpha-30 & \frac{120}{7} \leq \alpha<20 \\
15.925 \alpha-12 & 20 \leq \alpha<\frac{620}{3} \\
16.8 \alpha & \alpha \geq \frac{620}{3}\end{cases}\right.
$$

note that the marginal price is not increasing (for instance, around $\alpha=\frac{240}{17}$, it decreases from 15.925 to 15.075), whereas the average one is.

It is still true that the star-shaped price functional $\pi$ does not allow for convenient super-hedgings if and only if it is increasing.

Notably, if this is the case, instead of the set $G:=\{g: \mathcal{M} \rightarrow \mathbb{R}: g$ convex, $g \geqq \pi\}$ of Proposition 2 or the set $G_{0}:=\{g \in G: g(0)=0\}$ of (4), the subsets $G^{+}:=\{g: \mathcal{M} \rightarrow \mathbb{R}: g$ convex and increasing, $g \geqq \pi\}$ and $G_{0}^{+}:=\left\{g \in G^{+}: g(0)=0\right\}$ can be taken into consideration in order to represent $\pi$. Define indeed, for every 
$g \in G$, the functional $g^{+}: \mathcal{M} \rightarrow \mathbb{R}$ by $g^{+}(X):=\min \{g(Y): Y \geqq X\}:$ the fact that $g$ is convex ensures that such a minimum exists. It is clear that $g^{+}$is increasing and that $g^{+} \leqq g$; furthermore, it is possible to prove (by taking into consideration the monotonicity of $\pi$ ) that still $g^{+} \geqq \pi{ }^{11}$ This way, it is straightforward to realise that $\pi(X)=\min _{g \in G} g^{+}(X)$ and, therefore, that $\pi(X)=\min _{g \in G^{+}} g(X)$ for every $X \in \mathcal{M}$.

Since it is trivial that the minimum of a family of increasing functionals remains increasing, the above considerations allow us to conclude that a star-shaped functional does not allow for convenient super-hedgings if, and only if, it can be represented as the pointwise minimum of a family of increasing functionals. Note that this is quite analogous to what happened for convex and, before, for sublinear functionals: $\pi$ allows for no convenient super-hedgings if, and only if, all of the functionals in the representation of $\pi$ are increasing.

In order to deal with arbitrages, one more consideration is needed. Since, in the economical interpretation we gave, we are free to choose, among several agents, the best one to buy the pay-off $X$ we want to detain, it is reasonable to think that we may prefer to buy several different portfolios from the different agents, in such a way that the overall position matches or, better, super-hedges $X$. Mathematically speaking, we are dealing with the following object:

Definition 1. Let $\mathcal{X}$ be a real linear space, and $G:=\left\{g_{s}: \mathcal{X} \rightarrow \mathbb{R}: s \in S\right\}$ a family of real functionals on $\mathcal{X}$. The inf-convolution of $G$ is the functional $F: \mathcal{X} \rightarrow \mathbb{R} \cup\{-\infty,+\infty\}$ defined by, for every $X \in \mathcal{X}$,

$$
F(X):=\inf \left\{g_{s_{1}}\left(Z^{1}\right)+g_{s_{2}}\left(Z^{2}\right)+\cdots+g_{s_{n}}\left(Z^{n}\right): n \in \mathbb{N}, s_{1}, \cdots, s_{n} \in S, \sum_{j=1}^{n} Z^{j} \geqq X\right\} .
$$

This way, if we are given a set $\left\{\pi_{s}: s \in S\right\}$ of price functionals, each ideally corresponding to a different market (or agent), taking their inf-convolution amounts to buying a finite number of positions $Z^{1}, Z^{2}, \cdots, Z^{n}$, on the markets $s_{1}, s_{2}, \cdots, s_{n}$ respectively, in such a way that the overall pay-off $Z^{1}+Z^{2}+\cdots+Z^{n}$ super-hedges the desired pay-off $X$. Note that, as a consequence, the inf-convolution of a family of functionals always turns out to be smaller than their pointwise minimum. (Note also that, if the $g \in G$ are all increasing, the condition $Z^{1}+Z^{2}+\cdots+Z^{n} \geqq X \quad$ can be equivalently replaced by $Z^{1}+Z^{2}+\cdots+Z^{n}=X$.)

The following proposition holds true, with evident consequences from the financial point of view.

Proposition 3. Let $\mathcal{X}$ be a real linear space and $G:=\left\{g_{s}: \mathcal{X} \rightarrow \mathbb{R}: s \in S\right\}$ be a family of real functionals on $\mathcal{X}$ such that every $g \in G$ is increasing and such that $g(0)=0$. Let $F: \mathcal{X} \rightarrow \mathbb{R} \cup\{-\infty,+\infty\}$ be the infconvolution of $G$ : then $F$ is finite-valued, and

1. if every $g \in G$ is convex, then $F$ is convex;

2. if every $g \in G$ is star-shaped, then $F$ is star-shaped.

The fact is that, even if every $g \in G$ is such that $g(0)=0$, it is not necessary that $F(0)=0$. Economically speaking, even if the "original” markets do not allow either for arbitrages nor for convenient super- hedgings, a "cross-market” arbitrage may still be possible.

Example 8. Consider again the three markets of Example 7: we saw that $Y^{1}=\left[\begin{array}{l}300 \\ 300\end{array}\right]$ can be bought from the second agent at the price 276. It is immediate to check that for $-Y^{1}=\left[\begin{array}{l}-300 \\ -300\end{array}\right]$ it is $\pi_{1}\left(-Y^{1}\right)=-270$, $\pi_{2}\left(-Y^{1}\right)=-276$, and $\pi_{3}\left(-Y^{1}\right)=-306$ : therefore, $Y^{1}$ can be sold to the third agent for a gain of 306, thus making a "cross-market” arbitrage of 30.

As a consequence, the inf-convolution $\bar{\pi}$ of $\pi_{1}, \pi_{2}, \pi_{3}$ will be star-shaped and increasing, because of Proposition 3, but such that $\pi\left(\left[\begin{array}{l}0 \\ 0\end{array}\right]\right) \leq-30$. (The inequality is indeed strict: for instance,

\footnotetext{
${ }^{11}$ For every $X \in \mathcal{M}$, it is $g^{+}(X)=g(\bar{Y})$ for some $\bar{Y} \in \mathcal{M}, \quad \bar{Y} \geqq X$. It is $g(\bar{Y}) \geqq \pi(\bar{Y})$ because $g \in G$; therefore, by monotonicity of $\pi$, we get that $\pi(X) \leqq \pi(\bar{Y}) \leqq g(\bar{Y})=g^{+}(X)$.
} 
$\left[\begin{array}{l}180 \\ 140\end{array}\right]+\left[\begin{array}{l}120 \\ 160\end{array}\right]+\left[\begin{array}{l}-300 \\ -300\end{array}\right]=\left(\left[\begin{array}{l}0 \\ 0\end{array}\right]\right)$, and $\left.\pi_{1}\left(\left[\begin{array}{l}180 \\ 140\end{array}\right]\right)+\pi_{2}\left(\left[\begin{array}{l}120 \\ 160\end{array}\right]\right)+\pi_{3}\left(\left[\begin{array}{l}-300 \\ -300\end{array}\right]\right)=-33\right)$.

Although the technical details for a complete proof become too complex to be reported here, the key feature can be guessed from the following (and last) example.

Example 9. The function

$$
f: \mathbb{R} \rightarrow \mathbb{R}, \quad f(x):= \begin{cases}0.4 x & x<0 \\ 0.3 x & x \geq 0\end{cases}
$$

is increasing, star-shaped and such that $f(0)=0$. Yet, it is clear that $f(-10)=-4$ and $f(10)=3$, so that $f(-10)+f(10)=-1$. Note that the subdifferential of $f$ at 0 is the empty set (no straight line fits below the graph of $f)$.

The point that can be proven is that, if a star-shaped price functional is the result of a "best price" over several markets, "cross-market" arbitrages are possible if and only if there are assets whose price around 0 behaves like the function of Example 9. Therefore, the same condition seen for convex functionals applies: a star-shaped price functional does not allow for arbitrages, not even cross-market ones, if and only if the subdifferential $\Phi_{0}$ of $\pi$ at 0 contains at least a positive functional $\varphi_{0}$. If $\Phi_{0}=\varnothing$, of course such a condition is not satisfied, which immediately signals the possibility of arbitrages.

Note that cross-market convenient super-hedgings may still be possible, as Example 8 itself makes clear ${ }^{12}$ : as a consequence of Proposition 3, this is of course bound to happen every time that the price function is (starshaped but) not convex.

We can summarise the results of this section in the following:

Theorem 2. Let $\mathcal{M}$ be a linear space of financial assets, and $\pi: \mathcal{M} \rightarrow \mathbb{R}$ be a star-shaped pricing functional such that $\pi(0)=0$. Define $G:=\{g: \mathcal{M} \rightarrow \mathbb{R}: g$ convex, $g \geqq\} \pi$ and $G_{0}:=\{g \in G: g(0)=0\}$. Call $G^{+}:=\{g \in G: g$ increasing $\}$ and $G_{0}^{+}:=\left\{g \in G_{0}: g\right.$ increasing $\}=G^{+} \cap G_{0}$. Finally, call $F$ the inf-convolution of $G$ (see Definition 1) and define, as in Theorem 1, $\Phi_{0}:=\{\varphi: \mathcal{M} \rightarrow \mathbb{R}: \varphi$ linear, $\varphi \leqq \pi\}$. Then:

1. $G$ and $G_{0}$ are non-empty, closed and convex;

2. for every $X \in \mathcal{M}, \pi(X)=\min _{g \in G} g(X)=\min _{g \in G_{0}} g(X)$;

3. $\pi$ is increasing if and only if $\pi(X)=\min _{g \in G^{+}} g(X)=\min _{g \in G_{0}^{+}} g(X)$ for every $X \in \mathcal{M}$.

4. $F(0)=0$ is positive if and only if there exists a positive $\varphi \in \Phi_{0}$.

Remark 3. In this section, we have seen two ways to build the overall supply and demand function of a given asset in the case when several "markets" are available.

1. In the first case, seen at the beginning of the section, we supposed that the agent simply chooses the "best market”: implicitly, we imposed that every single trade can only happen with a single agent. In such a case, the market is chosen where the total price, or (which is the same) the average price, is the minimum one.

2. In the second case, by using the "inf-convolution" technique, we supposed that the agent is free to split the desired position into "chunks" and buy the various "chunks" separately on the various markets. This way, for every single additional unit, the agent chooses the market where the marginal price is the minimum one.

Depending on the type of price functionals on the "original" market, we saw that:

1. In the first case, when all of the markets feature either a convex or a star-shaped price functional, the overall price functional turns out to be star-shaped: we pointed indeed out that an increasing average price is obtained;

2. In the second case, the overall price functionals inherit the convexity or the star-shape of the original functions (namely, it is convex if all of the original pricing functionals are, and star-shaped likewise): the minimum marginal price is chosen, and yet it need not be increasing unless it is in the original markets already.

In some sense, we have found that convexity and star-shape are quite "stable" properties in financial markets.

Of course, several other "rules" can be imagined: for instance, some markets may be only available for pur-

${ }^{12}$ In Example 8, buying $\left[\begin{array}{l}180 \\ 140\end{array}\right]$ on the first market and $\left[\begin{array}{l}120 \\ 160\end{array}\right]$ on the second one costs less than buying $\left[\begin{array}{l}300 \\ 300\end{array}\right]$ on the cheapest ( i.e., still the second) one. 
chases, or for sales, or only some particular amounts can be bought (not just with a minimum or a maximum amount, as seen in the beginning of this section, but for instance for multiples of some "size" only), in such a way that the offer price function turns out not even to be star-shaped. Anyway, examining such cases goes beyond the scopes of the present paper.

\section{Analysis}

Four type of price systems, each a generalisation of the previous one, have been examined in this paper. From the last to the first, they are:

1. star-shaped prices:

$$
\pi(X)=\min _{g \in G} \max _{f_{g} \in L_{g}}\left[\varphi_{f_{g}}(X)+c_{f_{g}}\right] \quad\left(\text { with } c_{f_{g}} \leq 0\right) \text {; }
$$

2. convex (or granular) prices, obtained when $G$ is a singleton:

$$
\pi(X)=\max _{f \in L}\left[\varphi_{f}(X)+c_{f}\right] \quad\left(\text { with } c_{f} \leq 0\right) ;
$$

3. sublinear prices, obtained when ( $G$ is a singleton and) $c_{f}=0$ (and therefore $f=\varphi_{f}$ ) for every $f \in L$ :

$$
\pi(X)=\max _{\varphi \in L} \varphi(X)
$$

4. linear prices, obtained when ( $G$ is a singleton, $c_{f}=0$ for every $f \in L$, and furthermore) $L$ is a singleton:

$$
\pi(X)=\varphi(X) .
$$

The mentioned fact that both the pointwise minimum and the inf-convolution of a family of star-shaped functionals still are star-shaped seems to suggest that no further generalisation of this type should be fruitful.

For each of the above types, the conditions for absence of arbitrages and of convenient super-hedgings can be examined by taking into consideration the behaviour of the price functionals on the indicator functions $\mathbb{I}_{A}: \Omega \rightarrow \mathbb{R}$ with $A \subseteq \Omega$ (where, as usual, $\mathbb{I}_{A}(\omega)=1$ if $\omega \in A$ and $=0$ otherwise), which are all positive.

1. Linear prices: $\pi(X)=\varphi(X)$. The price of $\mathbb{I}_{A}$ is the value $\varphi\left(\mathbb{I}_{A}\right):$ it is then immediate that such a price is positive for every $A$ (i.e., that no arbitrages and no convenient super-hedgings are possible) if and only if $\varphi \geqq 0$. Intuitively, indeed, if $\varphi$ is not positive, then there exists an $A \subseteq \Omega^{13}$ such that $\varphi\left(\mathbb{I}_{A}\right)<0$, so that $\mathbb{I}_{A}$ makes an arbitrage.

2. Sublinear prices: $\pi(X)=\max _{\varphi \in L} \varphi(X)$, with $L=\{\varphi$ linear, $\varphi \leqq \pi\}$. Recall that the positivity of $\pi$ is equivalent to the positivity of its ask prices, and its (increasing) monotonicity is equivalent to the positivity of its bid prices. The ask price of $\mathbb{I}_{A}$ is $\pi_{a}\left(\mathbb{I}_{A}\right)=\pi\left(\mathbb{I}_{A}\right)=\max _{\varphi \in L} \varphi\left(\mathbb{I}_{A}\right)$ and its bid price is

$\pi_{b}\left(\mathbb{I}_{A}\right)=-\pi\left(-\mathbb{I}_{A}\right)=\min _{\varphi \in L} \varphi\left(\mathbb{I}_{A}\right):$ therefore, the ask price is positive as soon as one of the prices $\varphi\left(\mathbb{I}_{A}\right)$ is, i.e., if there exists a positive $\varphi \in L$, and the bid price is positive only if all of the prices $\varphi\left(\mathbb{I}_{A}\right)$ are, i.e., if all $\varphi \in L$ are positive. Intuitively, if there is a non-positive $\bar{\varphi} \in L$, then as above there exists an $A \subseteq \Omega$ such that $\bar{\varphi}\left(\mathbb{I}_{A}\right)<0$ : this means that $\pi_{b}\left(\mathbb{I}_{A}\right)<0$, so that $\mathbb{I}_{A}$ makes a convenient super-hedging of the null pay-off. Less intuitively, and indeed harder to prove, if all $\varphi \in L$ are not positive, then there exists an $\bar{A} \subseteq \Omega$ such that $\varphi(\bar{A})<0$ for every $\varphi \in L$ : in this case, $\pi_{a}\left(\mathbb{I}_{\bar{A}}\right)<0$, and $\mathbb{I}_{A}$ makes an arbitrage.

3. Convex prices: $\pi(X)=\max _{f \in L}\left[\varphi_{f}(X)+c_{f}\right]$, with $L=\{f$ affine, $f \leqq \pi\}$. Call $\Phi$ the set of all $\varphi_{f}$ such that $\left[\varphi_{f}+c_{f}\right] \in L$ for some $c_{f} \leq 0$. The ask price of $\mathbb{I}_{A}$ is positive as soon as $\bar{f}\left(\mathbb{I}_{A}\right)$ is: since $\bar{f}(0)=c_{\bar{f}} \leq 0$, this implies that $\varphi_{\bar{f}}$ is increasing, i.e., positive, that is to say, that there exists a positive $\varphi$ in $\Phi$.

As for arbitrages, since $\pi$ is not positively homogeneous, we need to take into consideration every $k \cdot \mathbb{I}_{A}$

\footnotetext{
${ }^{13}$ In the spirit of Remark 1, we should write $A \in \mathcal{F}$.
} 
for $k>0$ : in order to make an arbitrage, $f\left(\bar{k}_{A}\right)$ has to be negative for all $f \in L$ and for some $\bar{k}$. This happens if $\bar{k} \varphi_{f}\left(\mathbb{I}_{A}\right)+c_{f} \leq 0$ for every $f \in L$ : since this equality can be easily met when $c_{f}<0$ (it is enough to take $\bar{k}$ "small enough"), indeed only the $f \in L$ such that $c_{f}=0$ can be taken into consideration, and these $f$ s (which amount to the set $\Phi_{0}$ of the linear functionals of $L$ ) are precisely the ones that concur in determining the value of $\pi$ close to 0 . Therefore, the above arguments for sublinear functionals can be repeated in a "local" sense (i.e., for a $\bar{k} \mathbb{I}_{A}$ with $\bar{k}$ "small enough"), to conclude that $\pi$ does not allow for arbitrages if there exists a positive $\varphi \in \Phi_{0}$.

Intuitively, if there is a non-positive $\bar{\varphi} \in \Phi$, then as above there exists an $A \subseteq \Omega$ such that $\bar{\varphi}\left(\mathbb{I}_{A}\right)<0$ : since there is some $\bar{f} \in L$ such that $\bar{f}=\bar{\varphi}+\bar{c}$, it is possible to get also $\bar{f}\left(k \mathbb{I}_{A}\right)<0$ if $k$ is "big enough", and this means that $\pi_{b}\left(k \mathbb{I}_{A}\right)<0$ (convenient super-hedging). On the other hand, if all $\varphi \in \Phi_{0}$ are not positive, then there exists an $\bar{A} \subseteq \Omega$ such that $\varphi(\bar{A})<0$ for every $\varphi \in \Phi_{0}$ : in this case, it is possible to choose $k$ "small enough" so as to obtain $\pi_{a}\left(k \mathbb{I}_{\bar{A}}\right)<0$, and $k \mathbb{I}_{A}$ makes an arbitrage.

4. Star-shaped prices: $\pi(X)=\min _{g \in G} \max _{f_{g} \in L_{g}}\left[\varphi_{f_{g}}(X)+c_{f_{g}}\right]$. The ask price of $\mathbb{I}_{A}$ is positive only if all of the $g\left(\mathbb{I}_{A}\right)$ are positive for every convex $g \in G$ : in other words, $\pi$ does not allow for arbitrages only if all $g \in G$ do not allow for them. As for convenient super-hedgings, it is now quite complex to summarise the properties that have to be imposed on the convex functionals $g \in G$ in order to get an increasing functional (for instance, it is possible to prove that, for every $x \in \mathbb{R}, \min _{\alpha \in \mathbb{R}}\left[x^{2}+(2 \alpha-1) x+\alpha^{2}\right]=x$ : an increasing functional, i.e., the identity $x \rightarrow x$, is obtained as the pointwise minimum of a family of functions, none of which is increasing). We have nevertheless realized that, if $\pi$ is increasing, then only the increasing functionals $f \in L$ may be taken into consideration and, on the other hand, that the pointwise minimum of increasing functions is increasing: therefore, $\pi$ does not allow for arbitrages if it can be written as the minimum of a family of increasing convex functionals ${ }^{14}$. Intuitively, it is clear that an arbitrage is possible as soon as even a single market allows for it, but now, for convenient super-hedgings, things become quite different. Indeed, recall that a convenient super-hedging is a pair $X, Y \in \mathcal{M}$ such that $X \geqq Y$ but $\pi(X)<\pi(Y)$ : in order to "deactivate" such a situation, i.e., in order to obtain $\pi(Y) \leq \pi(X)$, it may be enough that on a single market $Y$ is sold at a cheaper price than $\pi(X)$.

It is clear that moving from linear to star-shaped price systems yields price systems whose properties are closer and closer to what happens in "real" financial markets. Yet, because of the fact that such systems are each the generalisation of the previous one, the "efficiency" conditions for each family of functionals propagates on the further generalisations in a reasonable way. All in all, therefore, these four price systems altogether make quite a versatile toolbox for building financial models, in the sense that, when a financial model is needed, it is easy to "fine tune" the level of precision to suit the needs of the considered problem.

\section{Conclusion}

A classical problem in Mathematical Finance is to study the prices of a suitable set of risky financial assets, modeled as random variables on some state sets, by means of suitable functionals defined on this set of random variables. The properties of the price functional reflect the assumptions on the market. In this paper, we analysed four types of price functionals. We first summarized the properties of the widely known linear functionals, obtained when the market was supposed to be perfect, and of sublinear functionals, which took into account the proportional frictions. Then, we introduced two more classes of functionals which accommodate a wider set of market frictions: granular (convex) functionals, obtained when the unit prices of traded assets were increasing

\footnotetext{
${ }^{14}$ To conclude the short example: in computing $\min _{a \in \mathbb{R}}\left[x^{2}+(2 \alpha-1) x+\alpha^{2}\right]$, for every $a \in \mathbb{R}$, instead of the functions

$f_{\alpha}(x)=x^{2}+(2 \alpha-1) x+\alpha^{2}$ it is possible to take into consideration the functions

$$
x \rightarrow f_{\alpha}^{+}(x)=\min \left\{f_{\alpha}(y): y \geq x\right\}= \begin{cases}\alpha-0.25 & x \leq \alpha-0.5 \\ x^{2}+(2 \alpha-1) x+\alpha^{2} & x>\alpha-0.5\end{cases}
$$
}

which are all convex and increasing. 
w.r.t. the traded amount, and star-shaped functionals, obtained when the average unit prices of traded assets were increasing w.r.t. the traded amount. We explored some characterizations of such functionals, together with their relationships with arbitrages and market inefficiencies, and performed a final analysis on their effectiveness in allowing for versatile modelling.

\section{References}

Artzner, P., Delbaen, F., Eber, J. M., \& Heath, D. (1999). Coherent Measures of Risk. Mathematical Finance, 9, $203-228$. http://dx.doi.org/10.1111/1467-9965.00068

Björk, T. (1999). Arbitrage Theory in Continuous Time. Oxford: Oxford University Press.

Black, F., \& Scholes, M. (1973). The Pricing of Options and Corporate Liabilities. Journal of Political Economy, 81, 637654. http://dx.doi.org/10.1086/260062

Castagnoli, E., Favero, G., \& Maccheroni, F. (2009). Marchetto, il prezzatore perfetto. Elogio dell'internalità. Milan: Electronic Draft.

Castagnoli, E., Favero, G., \& Modesti, P. (in print). Price Systems for Random Amounts: A Unified Approach. In D. Jakóbczak (Ed.), Analyzing Risk through Probabilistic Modeling in Operations Research. Hershey: IGI Global.

Castagnoli, E., Favero, G., \& Tebaldi, C. (2011). One-Penny Arbitrages, or: A Free Snack without a Free Lunch. Journal of Applied Computer Science \& Mathematics, 10, 20-21.

Cerreia-Vioglio, S., Maccheroni, F., Marinacci, M., \& Montrucchio, F. (2011). Risk Measures: Rationality and Diversification. Mathematical Finance, 21, 743-774.

Chateauneuf, A., \& Aouani, Z. (2008). Exact Capacities and Star-Shaped Distorted Probabilities. Mathematical Social Sciences, 56, 185-194. http://dx.doi.org/10.1016/j.mathsocsci.2008.01.006

Cvitanić, J., Pham, H., \& Touzi, N. (1999). A Closed-Form Solution for the Problem of Super-Replication under Transaction Costs. Finance and Stochastic, 3, 35-54. http://dx.doi.org/10.1007/s007800050051

Davis, M. H. A., \& Clark, J. M. C. (1994). A Note on Super-Replicating Strategies. Philosophical Transactions: Physical Sciences and Engineering, 347, 485-494.

de Finetti, B., \& Obry, S. (1933). L’optimum nella misura del riscatto. Atti del Secondo Congresso Nazionale di Scienza delle Assicurazioni, 2, 99-123.

Delbaen, F., \& Schachermayer, W. (1994). A General Version of the Fundamental Theorem of Asset Pricing. Mathematische Annalen, 300, 463-520. http://dx.doi.org/10.1007/BF01450498

Dothan, M. U. (1990). Prices in Financial Markets. Oxford: Oxford University Press.

El Karoui, N., \& Quenez, M. C. (1995). Dynamic Programming and Pricing of Contingent Claims in an Incomplete Market. SIAM Journal on Control and Optimization, 33, 29-66. http://dx.doi.org/10.1137/S0363012992232579

El Karoui, N., \& Ravanelli, C. (2009). Cash Sub-Additive Risk Measures and Interest Rate Ambiguity. Mathematical Finance, 19, 561-590. http://dx.doi.org/10.1111/j.1467-9965.2009.00380.x

Föllmer, H., \& Schied, A. (2002). Convex Measures of Risk and Trading Constraints. Finance and Stochastic, 6, $429-447$. http://dx.doi.org/10.1007/s007800200072

Hodges, S. D., \& Neuberger, A. (1989). Optimal Replication of Contingent Claims under Transaction Costs. The Review of Futures Markets, 8, 222-239.

Jouini, E. (1997). Market Imperfections, Equilibrium and Arbitrage. Lecture Notes in Mathematics, 1656, 247-307. http://dx.doi.org/10.1007/BFb0092002

Jouini, E., \& Kallal, H. (1995). Martingales and Arbitrage in Securities Markets with Transaction Costs. Journal of Economic Theory, 66, 178-197. http://dx.doi.org/10.1006/jeth.1995.1037

Koehl, P. F., \& Pham, H. (2000). Sublinear Price Functionals under Portfolio Constraints. Journal of Mathematical Economics, 33, 393-399. http://dx.doi.org/10.1016/S0304-4068(99)00024-5

Merton, R. (1973). Theory of Rational Option Pricing. Bell Journal of Economics and Management Science, 4, 141-183. http://dx.doi.org/10.2307/3003143

Pham, H., \& Touzi, N. (1999). The Fundamental Theorem of Asset Pricing with Cone Constraints. Annals of Mathematical Economics, 31, 265-279. http://dx.doi.org/10.1016/s0304-4068(97)00059-1

Pliska, S. (1997). Introduction to Mathematical Finance: Discrete Time Models (2nd ed.). Malden: Blackwell.

Rockafellar, R. T. (1970). Convex Analysis. Princeton: Princeton University Press. http://dx.doi.org/10.1515/9781400873173

Stewart, I., \& Tall, D. (1983). Complex Analysis. Cambridge: Cambridge University Press. 